\title{
Regularity and the behavior of eigenvalues for minimizers of a constrained $Q$-tensor energy for liquid crystals
}

\author{
Patricia Bauman $^{1}$ - Daniel Phillips ${ }^{1}$
}

Received: 3 November 2015 / Accepted: 28 May 2016/ Published online: 22 June 2016

(C) The Author(s) 2016. This article is published with open access at Springerlink.com

\begin{abstract}
We investigate minimizers defined on a bounded domain in $\mathbb{R}^{2}$ for the MaierSaupe Q-tensor energy used to characterize nematic liquid crystal configurations. The energy density is singular, as in Ball and Majumdar's modification of the Landau-de Gennes Qtensor model, so as to constrain the competing states to have eigenvalues in the closure of a physically realistic range. We prove that minimizers are regular and in several model problems we are able to use this regularity to prove that minimizers have eigenvalues strictly within the physical range.
\end{abstract}

\section{Mathematics Subject Classification 35J50}

\section{Introduction}

In this paper we prove regularity properties and bounds on the eigenvalues for local minimizers of an energy derived from Maier-Saupe theory, a model that is used to describe order in nematic liquid crystal materials (see [9].) We examine the special case of a liquid crystal occupying a cylindrical region in $\mathbb{R}^{3}$ with cross-section $\Omega \subset \mathbb{R}^{2}$, where $\Omega$ is an open bounded domain with a smooth $\left(C^{2}\right)$ boundary. The liquid crystal material is described at almost every $\left(x_{1}, x_{2}\right)$ in $\Omega$ by a $3 \times 3$ matrix $Q\left(x_{1}, x_{2}\right)$ and is assumed to be uniform in the $x_{3}$-direction. The function $Q$ is a tensor-valued order parameter, by which we mean that for almost every $\mathbf{x}=\left(x_{1}, x_{2}\right)$ in $\Omega$,

Communicated by J. Ball.

Research supported by NSF grant DMS-1412840.

$凶$ Daniel Phillips

phillips@purdue.edu

Patricia Bauman

baumanp@purdue.edu

1 Department of Mathematics, Purdue University, West Lafayette, IN 47907, USA 


$$
Q(\mathbf{x}) \in \mathcal{S}_{0}:=\left\{Q \in \mathbb{R}^{3 \times 3}: Q=Q^{t} \quad \text { and } \quad \operatorname{tr} Q=0\right\}
$$

where $\mathbb{R}^{3 \times 3}$ denotes the space of $3 \times 3$ real-valued matrices. The energy considered here is:

$$
\mathcal{F}(Q)=\int_{\Omega}\left[f_{e}(Q(\mathbf{x}), \nabla Q(\mathbf{x}))+f_{b}(Q(\mathbf{x}))\right] d \mathbf{x}
$$

To describe our assumptions on the energy density of $\mathcal{F}$, let $Q \in \mathcal{S}_{0}$ and let $\lambda_{1}(Q) \leq \lambda_{2}(Q) \leq$ $\lambda_{3}(Q)$ denote its eigenvalues. Define the set $\mathcal{M} \subset \mathcal{S}_{0}$ as the set of matrices $Q \in \mathcal{S}_{0}$ for which $\lambda_{i}(Q) \in\left(-\frac{1}{3}, \frac{2}{3}\right)$ for all $1 \leq i \leq 3$. Then $\mathcal{M}$ is an open, bounded, and convex subset of $\mathcal{S}_{0}$. Note that $\partial \mathcal{M}$ is the set of all $Q$ in $\mathcal{S}_{0}$ such that $\lambda_{i}(Q) \in\left[-\frac{1}{3}, \frac{2}{3}\right]$ for all $1 \leq i \leq 3$ and $\lambda_{j}(Q) \in\left\{-\frac{1}{3}, \frac{2}{3}\right\}$ for at least one $j$. We assume throughout the paper that $f_{b}$ satisfies

$$
f_{b}(Q)=\left\{\begin{array}{lll}
f(Q)-\kappa|Q|^{2}+b_{0} & \text { for } & Q \in \mathcal{M} \\
+\infty & \text { for } & Q \in \mathcal{S}_{0} \backslash \mathcal{M}
\end{array}\right.
$$

where $\kappa$ and $b_{0}$ are constants, $\kappa \geq 0, f$ is convex, $f \in C^{\infty}(\mathcal{M})$, and $\lim _{Q \rightarrow \partial \mathcal{M}} f(Q)=+\infty$. Such a function is bounded below and hence we assume without loss of generality that

$$
b_{0}=-\min _{Q \in \mathcal{M}}\left\{f(Q)-\kappa|Q|^{2}\right\}
$$

so that $f_{b} \geq 0$. Our assumptions on the bulk energy density $f_{b}$ are motivated by the work in the papers [2] by Ball and Majumdar and [7] by Katriel, Kventsel, Luckhurst, and Sluckin. In these papers a particular potential function $f(Q)=f_{m s}(Q)$ as described above was constructed consistent with Maier-Saupe theory. More precisely, it was proved in [2] and [7] that for each $Q \in \mathcal{M}$ there is a unique function $\hat{\rho}=\hat{\rho}(Q)$ in the set

$$
A_{Q}:=\left\{\rho \in L^{1}\left(\mathbb{S}^{2} ; \mathbb{R}\right): \rho \geq 0, \int_{\mathbb{S}^{2}} \rho(\mathbf{p}) d \mathbf{p}=1, Q=\int_{\mathbb{S}^{2}}\left(\mathbf{p} \otimes \mathbf{p}-\frac{1}{3} I\right) \rho(\mathbf{p}) d \mathbf{p}\right\}
$$

satisfying

$$
f_{m s}(Q):=\inf _{\rho \in A_{Q}} \int_{\mathbb{S}^{2}} \rho(\mathbf{p}) \ln (\rho(\mathbf{p})) d \mathbf{p}=\int_{\mathbb{S}^{2}} \hat{\rho}(\mathbf{p}) \ln (\hat{\rho}(\mathbf{p})) d \mathbf{p} .
$$

Given $Q \in \mathcal{M}$ the set $A_{Q}$ is the family of (absolutely continuous) probability densities $\rho$ with $Q$ as their normalized second moments. The set $A_{Q}$ is nonempty for $Q$ in $\mathcal{S}_{0}$ if and only if $Q \in \mathcal{M}$; if $Q \in \partial \mathcal{M}$ one can still represent it as the second moment of a density,

$$
Q=\int_{\mathbb{S}^{2}}\left(\mathbf{p} \otimes \mathbf{p}-\frac{1}{3} I\right) d \mu(\mathbf{p}),
$$

however in this case the density $\mu$ will be a singular measure and this is not considered physical. (See [2].) The densities in $A_{Q}$ then provide all possible physically admissible statistics for the local orientation of the liquid crystal molecules with second moments given by $Q$. It is proved in [2] and [7] that $f_{m s}(Q)$ has the properties postulated above for $f(Q)$ except for the fact that $f_{m s} \in C^{\infty}(\mathcal{M})$. This fact is proved in [5]. We next describe our assumptions on the elastic energy density $f_{e}$. Let 


$$
\begin{aligned}
\mathcal{D} & :=\left\{D=\left[D_{i j k}\right] 1 \leq i, j, k \leq 3:\right. \\
D_{i j k} & \left.=D_{j i k} \text { and } \sum_{\ell=1}^{3} D_{\ell \ell k}=0 \text { for each } i, j, \text { and } k\right\} .
\end{aligned}
$$

Note that $\mathcal{D}$ includes the tangent space for differentiable mappings $Q: \Sigma \rightarrow \mathcal{S}_{0}$ where $\Sigma$ is an open subset of $\mathbb{R}^{3}$, i.e., $\left[\partial_{x_{k}} Q_{i j}(\mathbf{x})\right] \in \mathcal{D}$ for each differentiable $\mathcal{S}_{0}$-valued $Q$ and each $\mathbf{x}$ in $\Sigma$. We assume that $f_{e}(Q, D)$ is continuous on $\overline{\mathcal{M}} \times \mathcal{D}$ and that there are constants $0<\alpha_{1} \leq \alpha_{2}<\infty, 0 \leq M_{1} \leq M_{2}<\infty$ so that

$$
\alpha_{1}|D|^{2}-M_{1} \leq f_{e}(Q, D) \leq \alpha_{2}|D|^{2}+M_{2}
$$

for all $(Q, D) \in \overline{\mathcal{M}} \times \mathcal{D}$. A specific class of elastic energy density functions that we have in mind are the Landau-de Gennes elastic energy densities $f_{l d}(Q, D)$ defined as polynomials in terms of the components of $Q$ and $D$ that are $S O(3)$ invariant, that is, $f_{l d}(Q, D)=$ $f_{l d}\left(Q^{*}, D^{*}\right)$ for every $(Q, D)$ in $\mathcal{S}_{0} \times \mathcal{D}$ and $R$ in $S O(3)$ where

$$
\begin{aligned}
Q_{i j}^{*} & =R_{i \ell} Q_{\ell m} R_{j m}, \\
D_{i j k}^{*} & =R_{i \ell} R_{j m} R_{k h} D_{\ell m h} .
\end{aligned}
$$

Here we used the summation convention for repeated indices among $\ell, m, h$ in the set $\{1,2,3\}$. We remark that for the case of a Landau-de Gennes elastic energy density which is a function of $D$ only, denoted by $f_{l d}(Q, D)=f_{l d}^{(1)}(D)$, it is known that $f_{l d}^{(1)}$ satisfies (1.4) if and only if for any differentiable function $Q\left(x_{1}, x_{2}, x_{3}\right)$ valued in $\mathcal{S}_{0}$ we have

$$
\begin{aligned}
f_{l d}^{(1)}(\nabla Q)= & L_{1} Q_{i j, x_{\ell}} Q_{i j, x_{\ell}}+L_{2} Q_{i j, x_{j}} Q_{i k, x_{k}} \\
& +L_{3} Q_{i j, x_{k}} Q_{i k, x_{j}}
\end{aligned}
$$

with the elasticity constants satisfying

$$
L_{1}+\frac{5}{3} L_{2}+\frac{1}{6} L_{3}>0, L_{1}-\frac{1}{2} L_{3}>0, L_{1}+L_{3}>0 .
$$

(See [8].) This is a classic example among Landau-de Gennes models. More general examples of Landau de Gennes energy densities are also given in [8] such as

$$
\begin{aligned}
f_{l d}^{(2)}(Q, \nabla Q)= & f_{l d}^{(1)}(\nabla Q)+L_{4} \varepsilon_{l k j} Q_{l i} Q_{k i, x_{j}} \\
& +L_{5} Q_{l k} Q_{i j, x_{l}} Q_{i j, x_{k}}
\end{aligned}
$$

where $\varepsilon_{\ell k j}$ is the Levi-Civita tensor. The $L_{4}$ term above allows the model to account for molecular chirality, and cubic expressions such as the $L_{5}$ term permit a more specific description of elastic contributions. Since $\overline{\mathcal{M}}$ is a bounded set one can easily give conditions on the elasticity constants so that $f_{l d}^{(2)}$ satisfies (1.4). [See (1.10).] We say that $Q$ is in $H_{l o c}^{1}(\Omega ; \overline{\mathcal{M}})$ if and only if $Q$ is in $H_{l o c}^{1}\left(\Omega ; \mathbb{R}^{3 \times 3}\right)$ and that $Q(\mathbf{x}) \in \overline{\mathcal{M}}$ for almost every $\mathbf{x} \in \Omega$. Note that in this paper we apply the energy density $f_{l d}(Q, D)$ to functions $Q$ in $H_{l o c}^{1}(\Omega ; \overline{\mathcal{M}})$ where $\Omega$ is a subset of the $x_{1} x_{2}$ plane, and as such for the functions considered here we have $D_{i j 3}=Q_{i j, x_{3}}=0$. We consider fixed boundary conditions,

$$
Q=Q_{0} \in C^{0,1}(\partial \Omega ; \overline{\mathcal{M}}) \text { such that } \int_{\partial \Omega} f_{b}\left(Q_{0}\right) d s<\infty .
$$

Set

$$
\mathcal{A}_{0}=\left\{Q \in H^{1}(\Omega ; \overline{\mathcal{M}}): Q=Q_{0} \text { on } \partial \Omega\right\} .
$$


One can show that there exists $Q \in \mathcal{A}_{0}$ such that $\mathcal{F}(Q)<\infty$.

In this paper, under the assumptions described above on $\Omega, f_{e}, f_{b}$, and $Q_{0}$, we analyze finite energy local minimizers of (1.1) and global minimizers in $\mathcal{A}_{0}$. Note that a Landau-de Gennes elastic energy density $f_{e}=f_{l d}(Q, D)$ satisfies (1.4) if and only if it is of degree two in $D$ for each $Q$ and the mapping $D \in \mathcal{D} \rightarrow f_{l d}(Q, D)$ is strictly convex, uniformly in $Q$ for $Q \in \mathcal{M}$. In light of this, if $f_{e}=f_{l d}$ the existence of global minimizers in $\mathcal{A}_{0}$ follows in a standard way by using direct methods, just as in [2].

Our main results can be described as follows. In Sect. 2 we first prove that local minimizers of $\mathcal{F}$ in $H^{1}(\Omega ; \overline{\mathcal{M}})$ are uniformly Hölder continuous in subdomains whose closure is a compact subset of $\Omega$. This follows immediately from:

Theorem 1 Assume $r_{0}>0,0<r<r_{0}$, and $B_{4 r}(\mathbf{o})$ is an open disk contained in $\Omega$ with center $\mathbf{0}$ and radius $4 r$. Let $B_{S}=B_{S}(\mathbf{o})$. Assume $Q \in H^{1}\left(B_{4 r} ; \overline{\mathcal{M}}\right)$ such that

$$
\mathcal{F}\left(Q ; B_{4 r}\right):=\int_{B_{4 r}}\left[f_{e}(Q, \nabla Q)+f_{b}(Q)\right] d \mathbf{x}<\infty
$$

and

$$
\mathcal{F}\left(Q ; B_{4 r}\right) \leq \mathcal{F}\left(V ; B_{4 r}\right)
$$

for all $V \in H^{1}\left(B_{4 r} ; \overline{\mathcal{M}}\right)$ such that $V-Q \in H_{0}^{1}\left(B_{4 r} ; \mathcal{S}_{0}\right)$. Then there exists a constant $\sigma$ in $(0,1)$ and $c>0$ depending only on $r_{0}$ and on the constants in (1.2) and (1.4) such that $Q \in C^{\sigma}\left(\bar{B}_{r}\right)$ and

$$
\|Q\|_{C^{\sigma}\left(\bar{B}_{r}\right)} \leq \frac{c}{r^{\sigma}} \sqrt{\left(\mathcal{F}\left(Q ; B_{2 r}\right)+1\right)} .
$$

We then consider local minimizers near $\partial \Omega$. Let $r_{1}$ be a positive constant such that for each point $\mathbf{o}$ on $\partial \Omega, B_{4 r_{1}}(\mathbf{o}) \cap \Omega$ is a coordinate neighborhood for the $C^{2}$ structure of $\partial \Omega$ and is diffeomorphic to a half-disk.

Theorem 2 Assume $\mathbf{0}$ is any point in $\partial \Omega$ and let $B_{S}=B_{S}(\mathbf{0})$. There exists a constant $\sigma$ in $(0,1)$ and $c>0$ depending only on $\Omega, r_{1}, Q_{0}$, and the constants in (1.2) and (1.4), so that if $0<r \leq r_{1}, \quad Q \in H^{1}\left(\Omega \cap B_{4 r} ; \overline{\mathcal{M}}\right), \quad Q=Q_{0}$ on $B_{4 r} \cap \partial \Omega, \quad \mathcal{F}\left(Q ; \Omega \cap B_{4 r}\right)<\infty$, and

$$
\mathcal{F}\left(Q ; \Omega \cap B_{4 r}\right) \leq \mathcal{F}\left(V ; \Omega \cap B_{4 r}\right)
$$

for all $V \in H^{1}\left(\Omega \cap B_{4 r} ; \overline{\mathcal{M}}\right)$ such that $V-Q \in H_{0}^{1}\left(\Omega \cap B_{4 r} ; \mathcal{S}_{0}\right)$, then $Q \in C^{\sigma}\left(\overline{\Omega \cap B_{r}}\right)$ and

$$
\|Q\|_{C^{\sigma}\left(\overline{\Omega \cap B_{r}}\right)} \leq \frac{c}{r^{\sigma}} \sqrt{\left(\mathcal{F}\left(Q ; B_{2 r}\right)+1\right)} .
$$

In Maier-Saupe theory the set

$$
\begin{aligned}
\Lambda(Q)= & \{\mathbf{x} \in \bar{\Omega}: Q(\mathbf{x}) \in \partial \mathcal{M}\} \\
\equiv & \left\{\mathbf{x} \in \bar{\Omega}: \lambda_{i}(Q(\mathbf{x})) \in\left[-\frac{1}{3}, \frac{2}{3}\right] \text { for } 1 \leq i \leq 3\right. \text { and } \\
& \left.\lambda_{j}(Q(\mathbf{x})) \in\left\{-\frac{1}{3}, \frac{2}{3}\right\} \text { for some } j \in\{1,2,3\}\right\}
\end{aligned}
$$

corresponds to locations where perfect nematic order occurs and this is interpreted as not physical. (See [2].) Note that by (1.2), if $\mathcal{F}(Q ; E)<\infty$ then $\Lambda(Q) \cap E$ has measure zero. Thus we have: 
Corollary 1 Let $Q$ be a minimizer of $\mathcal{F}$ in $\mathcal{A}_{0}$. Then $Q$ is Hölder continuous in $\bar{\Omega}$ and hence $\Lambda(Q)$ is compact.

We next assume that $f_{e}=f_{l d}(Q, D)$ [(and hence from our assumptions $f_{l d}$ satisfies (1.4)]. The condition $Q \in \mathcal{S}_{0}$ allows us to express the nine components of $Q$ in terms of the five independent variables

$$
\mathbf{z}=\left(z^{1}, \ldots, z^{5}\right)=\left(Q_{11}, Q_{12}, Q_{13}, Q_{22}, Q_{23}\right),
$$

as $Q=\tilde{Q}(\mathbf{z})$, so that $Q(\mathbf{x})=\tilde{Q}(\mathbf{z}(\mathbf{x}))$. The energy takes the form

$$
\begin{aligned}
\mathcal{F}^{\prime}(\mathbf{z})= & \int_{\Omega}\left[a_{i j}^{l k}(\mathbf{z}) \partial_{x_{l}} z^{i} \partial_{x_{k}} z^{j}+b_{i}^{\ell}(\mathbf{z}) \partial_{x_{l}} z^{i}\right. \\
& \left.+f_{b}(\tilde{Q}(\mathbf{z}))\right] d \mathbf{x}
\end{aligned}
$$

where the coefficients are polynomials and there is a constant $\lambda>0$ so that

$$
\begin{aligned}
& a_{i j}^{l k}(\mathbf{z}) \zeta_{l}^{i} \zeta_{k}^{j} \geq \lambda|\zeta|^{2} \text { for all } \\
& \zeta \in \mathbb{R}^{5 \times 2} \text { and } \mathbf{z} \text { such that } \tilde{Q}(\mathbf{z}) \in \overline{\mathcal{M}}
\end{aligned}
$$

From elliptic regularity theory (see $[6,10])$ we obtain the following:

Corollary 2 Assume $f_{e}=f_{l d}(Q, D)$ and $f_{l d}$ satisfies our assumptions. Then:

1. If $B$ is an open disk contained in $\Omega$, a finite energy local minimizer $Q$ in $H^{1}(B ; \overline{\mathcal{M}})$ for $\mathcal{F}$ satisfies $Q \in C^{\infty}(B \backslash \Lambda(Q))$.

2. If $Q$ and $B=B_{4 r}(\mathbf{o})$ are as in Theorem 2 such that $B \cap \Lambda(Q)=\emptyset$, then $Q$ is as smooth in $B_{r}(\mathbf{o}) \cap \bar{\Omega}$ as $\partial \Omega$ and $Q_{0}$ allow. In particular if $B \cap \partial \Omega$ is of class $C^{k, \alpha}$ and $Q_{0} \in C^{k, \alpha}(B \cap \partial \Omega)$ for some $k \geq 2$ and $0<\alpha<1$, then $Q \in C^{k, \alpha}\left(B_{r}(\mathbf{o}) \cap \bar{\Omega}\right)$.

In Sect. 3 we analyze $\Lambda(Q)$ for local minimizers, in the case

$$
\begin{aligned}
f_{e}(Q, \nabla Q)= & L_{1}|\nabla Q|^{2}+L_{4} \varepsilon_{l k j} Q_{\ell i} Q_{k i, x_{j}} \\
& +L_{5} Q_{l k} Q_{i j, x_{l}} Q_{i j, x_{k}} .
\end{aligned}
$$

If $(Q, D) \in \overline{\mathcal{M}} \times \mathcal{D}$, we have

$$
-\frac{1}{3}|D|^{2} \leq Q_{l k} D_{i j l} D_{i j k} \leq \frac{2}{3}|D|^{2} .
$$

Thus the natural ellipticity condition for (1.9) is

$$
\begin{array}{ll}
L_{1}-\frac{L_{5}}{3}>0 & \text { if } L_{5} \geq 0 \\
L_{1}+\frac{2 L_{5}}{3}>0 & \text { if } L_{5}<0 .
\end{array}
$$

It follows that if $L_{1}$ and $L_{5}$ satisfy (1.10) and $L_{4}$ is fixed then (1.4) holds for the elastic density (1.9) for some positive constants $\alpha_{1}, \alpha_{2}, M_{1}, M_{2}$. The uniform convexity of the elastic density $f_{e}(Q, D)$ in $D$ is due to having $Q$ constrained to values in $\overline{\mathcal{M}}$. This structure leads to the existence of minimizers for $\mathcal{F}$ over $\mathcal{A}_{0}$ with $f_{e}$ as in (1.9) using direct methods. In contrast to this setting, in [2] Ball and Majumdar examined the classical unconstrained (Landau-de Gennes) energy that included the case where $f_{e}$ is as in (1.9) and $f_{b}$ is a polynomial in $Q$. They studied the problem of minimizing the energy over $H^{1}\left(\Omega ; \mathcal{S}_{0}\right)$ with $Q=Q_{0}$ on $\partial \Omega$. They proved that if $L_{5} \neq 0$ then the energy is not bounded below and the unconstrained minimum 
problem is ill-posed. Thus the unconstrained energy is ill suited for investigating certain relevant elastic effects. These observations motivate investigating the minimum problem for the Maier-Saupe energy. Minimizers for the constrained problem, however, may a priori take on un-physical states. We show that if $f_{e}$ is as in (1.9) then this is impossible. We prove the following physicality result.

Theorem 3 Let $Q$ be a finite energy local minimizer for $\mathcal{F}(\cdot ; B)$ for a ball $B \subset \Omega$ where $f_{e}$ is as in (1.9) satisfying (1.10). Then $\Lambda(Q) \cap B=\emptyset$ and we have $Q \in C^{\infty}(B)$.

Theorem 3 generalizes a result of Ball and Majumdar [2] proved for the case in which $\Omega$ is replaced by a domain $D$ in $\mathbb{R}^{3}$, the elasticity density $f_{e}(\nabla Q)=L_{1}|\nabla Q|^{2}$, and $Q_{0}$ is valued in a compact subset of $\mathcal{M}$. They proved that minimizers $Q$ in $\mathcal{A}_{0}$ are in $C^{\infty}(D)$ and have $\Lambda(Q)=\emptyset$. In this paper we extend their result to minimizers and local minimizers defined on a domain $\Omega \subset \mathbb{R}^{n}$ based on our approach. We work with a slightly more general setting than used above. Let $\mathcal{K} \subset \mathbb{R}^{m}$ be an open, bounded, convex set where $m \geq 1$. Consider $\tilde{f}: \mathcal{K} \rightarrow \mathbb{R}$ given as $\tilde{f}(\mathbf{p})=f(\mathbf{p})-\kappa|\mathbf{p}|^{2}$ where $\kappa \geq 0, f \geq 0, f \in C^{\infty}(\overline{\mathcal{K}})$ and convex such that $\lim _{\mathbf{p} \rightarrow \partial \mathcal{K}} f(\mathbf{p})=\infty$.

Theorem 4 Let $\Omega$ be a domain in $\mathbb{R}^{n}$ for $n \geq 1, \gamma>0$, and let $u(\mathbf{x}) \in H^{1}(\Omega ; \overline{\mathcal{K}})$ be a local minimizer for

$$
\mathcal{F}_{1}(w)=\int_{\Omega}\left[\gamma|\nabla w|^{2}+\tilde{f}(w)\right] d \mathbf{x}
$$

such that $\mathcal{F}_{1}(u)<\infty$. Then $u(\Omega) \subset \mathcal{K}, u \in C^{\infty}(\Omega)$, and $u$ is a solution to

$$
2 \gamma \Delta u(\mathbf{x})=D_{u} \tilde{f}(u(\mathbf{x})) \text { in } \Omega .
$$

Furthermore if $\partial \Omega$ is of class $C^{2, \sigma}$ for some $0<\sigma<1$ and $u$ takes on boundary values $u=u_{0} \in C^{\sigma}(\partial \Omega ; \mathcal{K})$, then $u(\Omega) \subset \subset \mathcal{K}$ and it follows that $u \in C^{\sigma}(\bar{\Omega} ; \mathcal{K})$.

Applications of Theorem 4 to the Maier-Saupe energy functional are given in Corollaries 3 and 4. Further prior work concerning regularity results for the constrained minimum problem has been done by Evans et al. [4] where they investigated minimizers for energies of the form (1.1) assuming that $\Omega \subset \mathbb{R}^{n}$ for $n \geq 2$ and proved partial regularity results.

\section{Hölder continuity and higher regularity}

Our principal technique is to use elliptic replacements as a way of constructing comparison functions valued in $\overline{\mathcal{M}}$.

Definition 2.1 Let $Q \in H^{1}(\Omega ; \overline{\mathcal{M}})$ and let $B$ be an open ball in $\mathbb{R}^{2}$ such that $B \cap \Omega \neq \emptyset$. Set

$$
Q_{B}(\mathbf{x})= \begin{cases}Q(\mathbf{x}), & \text { for } \quad \mathbf{x} \in \Omega \backslash B, \\ H(\mathbf{x})=\left[H_{i j}(\mathbf{x})\right], & \text { for } \quad \mathbf{x} \in B \cap \Omega \text { and } 1 \leq i, j \leq 3,\end{cases}
$$

where

$$
\begin{aligned}
\Delta H_{i j} & =0 \quad \text { in } B \cap \Omega, \\
H_{i j} & =Q_{i j} \quad \text { on } \partial(B \cap \Omega) .
\end{aligned}
$$

Lemma 2.1 $Q_{B}(\mathbf{x}) \in \overline{\mathcal{M}}$ for all $\mathbf{x} \in \Omega$. In addition, $Q_{B}\left(\mathbf{x}_{0}\right) \in \partial \mathcal{M}$ for some $\mathbf{x}_{0} \in B \cap \Omega$ if and only if $Q_{B}(\mathbf{x}) \in \partial \mathcal{M}$ for all $\mathbf{x}$ in the component of $B \cap \Omega$ containing $\mathbf{x}_{0}$. 
Proof Let $\operatorname{tr} A$ denote the trace of the square matrix $A$. We have that $\operatorname{tr} Q_{B}(\mathbf{x})=0$ and $Q_{B}(\mathbf{x})=Q_{B}^{t}(\mathbf{x})$ in $H^{1 / 2}(\partial[B \cap \Omega])$. Thus these equalities hold for all $\mathbf{x} \in B \cap \Omega$ since $Q_{B}$ is harmonic in $B \cap \Omega$. It follows that $Q_{B}(\mathbf{x}) \in \mathcal{S}_{0}$ for all $\mathbf{x} \in B \cap \Omega$. Next we define

$$
g(\mathbf{x} ; \zeta)=\zeta^{t} Q_{B}(\mathbf{x}) \zeta \text { for each } \mathbf{x} \in B \cap \Omega \text { and } \zeta \in \mathbb{R}^{3},|\zeta|=1 .
$$

For each $\zeta$ this is a harmonic function in $\mathbf{x}$. We have that $Q\left(\mathbf{x}_{0}\right) \in \partial \mathcal{M}$ for some $\mathbf{x}_{0} \in B \cap \Omega$ if and only if $g\left(\mathbf{x}_{0} ; \zeta_{0}\right)=\frac{2}{3}$ or $-\frac{1}{3}$ for some $\zeta_{0}$. Since $-\frac{1}{3} \leq g\left(\mathbf{x} ; \zeta_{0}\right) \leq \frac{2}{3}$ then by the strong maximum principle such an $\mathbf{x}_{0}$ exists if and only if $Q_{B}(\mathbf{x}) \in \partial \mathcal{M}$ for all $\mathbf{x}$ in the component of $B \cap \Omega$ containing $\mathbf{x}_{0}$.

Recall that if $D$ is an open subset of $\mathbb{R}^{n}$ with a piece-wise smooth boundary then a function $v \in H^{1}(D)$ is defined pointwise $\mathcal{H}^{n-1}$-almost everywhere on $\bar{D}$. (See [3].)

Definition 2.2 Let $U$ be an open set in $\mathbb{R}^{n}$ with a piecewise smooth boundary and let $E \subset \bar{U}$ be an $\mathcal{H}^{n-1}$-measurable set. A function $Q \in H^{1}(U ; \overline{\mathcal{M}})$ is defined to be separated from $\partial \mathcal{M}$ on $E$ if and only if ess inf $\operatorname{dist}(Q(\mathbf{x}), \partial \mathcal{M})>0$ where the essential infimum is taken with respect to $\mathcal{H}^{n-1}$-measure.

Proof of Theorem 1 Let $\mathbf{x}_{0} \in B_{r}(\mathbf{o}), 0<\rho \leq r, \frac{\rho}{2} \leq s \leq \rho$, and set $Q_{s}(\mathbf{x}):=Q_{B_{s}\left(\mathbf{x}_{0}\right)}(\mathbf{x})$. By (1.2), $f_{b}\left(Q_{s}\right)=f_{b}(H) \leq f(H)+b_{0}$ in $B_{s}\left(\mathbf{x}_{0}\right)$. Using (1.4) and the fact that $|Q|$ is uniformly bounded almost everywhere in $B_{4 r}(\mathbf{o})$ (since $Q$ is valued in $\overline{\mathcal{M}}$ a.e.) we have

$$
\begin{aligned}
\int_{B_{\rho / 2}\left(\mathbf{x}_{0}\right)}\left[\alpha_{1}|\nabla Q|^{2}+f_{b}(Q)\right] d \mathbf{x} \leq & \mathcal{F}\left(Q ; B_{2 \rho}\left(\mathbf{x}_{0}\right)\right)+C_{0} \rho^{2} \\
\leq & \mathcal{F}\left(Q_{s} ; B_{2 \rho}\left(\mathbf{x}_{0}\right)\right)+C_{0} \rho^{2} \\
= & \mathcal{F}\left(Q_{s} ; B_{s}\left(\mathbf{x}_{0}\right)\right)+\mathcal{F}\left(Q ; B_{2 \rho}\left(\mathbf{x}_{0}\right) \backslash B_{S}\left(\mathbf{x}_{0}\right)\right)+C_{0} \rho^{2} \\
\leq & \alpha_{2} \int_{B_{s}\left(\mathbf{x}_{0}\right)}|\nabla H|^{2} d \mathbf{x}+\int_{B_{s}\left(\mathbf{x}_{0}\right)} f(H) d \mathbf{x} \\
& +\mathcal{F}\left(Q ; B_{2 \rho}\left(\mathbf{x}_{0}\right) \backslash B_{S}\left(\mathbf{x}_{0}\right)\right)+C_{1} \rho^{2}
\end{aligned}
$$

where $C_{1}$ depends only on $M_{1}, M_{2}$, and $b_{0}$. Let $\bar{Q}$ be the average of $Q$ on $B_{2 \rho}\left(\mathbf{x}_{0}\right) \backslash B_{S}\left(\mathbf{x}_{0}\right)$. Since $H$ is harmonic and $H=Q$ on $\partial B_{S}\left(\mathbf{x}_{0}\right)$ we have

$$
\begin{aligned}
\int_{B_{s}\left(\mathbf{x}_{0}\right)}|\nabla H|^{2} d \mathbf{x} & =\int_{B_{S}\left(\mathbf{x}_{0}\right)}|\nabla(H-\bar{Q})|^{2} d \mathbf{x} \\
& \leq C_{2}\|Q-\bar{Q}\|_{H^{1 / 2}\left(\partial B_{s}\left(\mathbf{x}_{0}\right)\right)}^{2} \\
& \leq C_{3}\|Q-\bar{Q}\|_{H^{1}\left(B_{2 \rho}\left(\mathbf{x}_{0}\right) \backslash B_{S}\left(\mathbf{x}_{0}\right)\right)}^{2} \\
& \leq C_{4} \int_{B_{2 \rho}\left(\mathbf{x}_{0}\right) \backslash B_{S}\left(\mathbf{x}_{0}\right)}\left(|\nabla Q|^{2}+\rho^{-2}|Q-\bar{Q}|^{2}\right) d \mathbf{x} \\
& \leq C_{5} \int_{B_{2 \rho}\left(\mathbf{x}_{0}\right) \backslash B_{S}\left(\mathbf{x}_{0}\right)}|\nabla Q|^{2} d \mathbf{x}
\end{aligned}
$$

where the $C_{i}$ are independent of $s, \rho$, and $Q$ for $\frac{\rho}{2} \leq s \leq \rho$. We next take $s$ so that

$$
\int_{\partial B_{S}\left(\mathbf{x}_{0}\right)} f_{b}(Q) d \mathbf{s}<\infty
$$

where $d$ s denotes arc-length. This holds for almost every $\frac{\rho}{2} \leq s \leq \rho$. Note that (2.3) holds if and only if $Q(\mathbf{x}) \in \mathcal{M}$ almost everywhere in $\partial B_{s}\left(\mathbf{x}_{0}\right)$ and $f(Q) \in L^{1}\left(\partial B_{S}\left(\mathbf{x}_{0}\right)\right)$. If $Q$ is 
separated from $\partial \mathcal{M}$ on $\partial B_{S}\left(\mathbf{x}_{0}\right)$ then $f$ is $C^{\infty}$ on a neighborhood of the range of $\left.H\right|_{B_{S}\left(\mathbf{x}_{0}\right)}$. Let $Q=\tilde{Q}(\mathbf{z})$ as in (1.6). It follows that $f(\tilde{Q}(\mathbf{z}))$ is a convex function of $\mathbf{z}$ and that is a smooth function for all $\mathbf{z}$ such that $\tilde{Q}(\mathbf{z})$ is in this neighborhood. Thus $f(H(\mathbf{x}))=f(\tilde{Q}(\mathbf{z}(\mathbf{x})))$, with $\mathbf{z}(\mathbf{x})=\left(z_{1}, \ldots, z_{5}\right)(\mathbf{x})=\left(H_{11}, H_{12}, H_{13}, H_{22}, H_{23}\right)(\mathbf{x})$, is a classical subharmonic function on $B_{S}\left(\mathbf{x}_{0}\right)$. Indeed

$$
\begin{aligned}
\Delta f(H(\mathbf{x})) & =\Delta[f(\tilde{Q}(\mathbf{z}(\mathbf{x})))] \\
& =\partial_{z_{l}}\left[\left.f(\tilde{Q}(\mathbf{z})]\right|_{\mathbf{z}(\mathbf{x})} \Delta z_{l}(\mathbf{x})+\left.\partial_{z_{l} z_{k}}^{2} f(\tilde{Q}(\mathbf{z}))\right|_{\mathbf{z}(\mathbf{x})} \partial_{x_{i}} z_{l} \partial_{x_{i}} z_{k} \geq 0 .\right.
\end{aligned}
$$

It follows from this and the mean value theorem that

$$
\int_{B_{S}\left(\mathbf{x}_{0}\right)} f(H) d \mathbf{x} \leq \frac{s}{2} \int_{\partial B_{S}\left(\mathbf{x}_{0}\right)} f(Q) d \mathbf{s} .
$$

If $Q$ is not separated from $\partial \mathcal{M}$ on $\partial B_{S}\left(\mathbf{x}_{0}\right)$ we consider $\tau H(\mathbf{x})$ for $\tau \uparrow 1$ and $\mathbf{x} \in B_{S}\left(\mathbf{x}_{0}\right)$. By the maximum principle, since $H=Q$ on $\partial B_{S}\left(\mathbf{x}_{0}\right)$, we have for $0<\tau<1$ that

$$
-\frac{1}{3}<\frac{-\tau}{3} \leq \tau \inf _{\substack{\mathbf{x} \in B_{S}\left(\mathbf{x}_{0}\right) \\|\zeta|=1}} \zeta^{t} H(\mathbf{x}) \zeta \leq \tau \sup _{\substack{\mathbf{x} \in B_{s}\left(\mathbf{x}_{0}\right) \\|\zeta|=1}} \zeta^{t} H(\mathbf{x}) \zeta \leq \frac{2 \tau}{3}<\frac{2}{3} .
$$

Thus $\tau H \in \mathcal{M}$ on $B_{S}\left(\mathbf{x}_{0}\right), \tau Q \in \mathcal{M}$ almost everywhere on $\partial B_{S}\left(\mathbf{x}_{0}\right)$, and $f(\tau Q) \in$ $L^{1}\left(\partial B_{S}\left(\mathbf{x}_{0}\right)\right)$ for each $0<\tau<1$, and we get

$$
\int_{B_{S}\left(\mathbf{x}_{0}\right)} f(\tau H) d \mathbf{x} \leq \frac{s}{2} \int_{\partial B_{S}\left(\mathbf{x}_{0}\right)} f(\tau Q) d \mathbf{s} .
$$

Note that since $f$ is convex we have

$$
f(\tau Q) \leq \tau f(Q)+(1-\tau) f(O) \text { for all } Q \in \mathcal{M} .
$$

As $f$ is bounded below on $\mathcal{M}$ we can apply the dominated converges theorem to the right side and Fatou's lemma to the left as $\tau \uparrow 1$. Thus (2.4) holds for all $s$ such that (2.3) is true. Next choose a constant $C_{6}=C_{6}\left(b_{0}, \kappa\right)$ and a value $\frac{\rho}{2} \leq \bar{s} \leq \rho$ so that

$$
\begin{aligned}
\frac{\bar{s}}{2} \int_{\partial B_{\bar{S}}\left(\mathbf{x}_{0}\right)} f(Q) d \mathbf{s} & \leq \frac{\bar{s}}{2} \int_{\partial B_{\bar{S}}\left(\mathbf{x}_{0}\right)} f_{b}(Q) d \mathbf{s}+C_{6} \rho^{2} \\
& \leq \int_{B_{\rho}\left(\mathbf{x}_{0}\right) \backslash B_{\rho / 2}\left(\mathbf{x}_{0}\right)} f_{b}(Q) d \mathbf{x}+C_{6} \rho^{2} .
\end{aligned}
$$

Set $s=\bar{s}$ in (2.2) and (2.4); using these statements with (2.1) and (2.5) we arrive at

$$
\begin{aligned}
\mu_{1} \int_{B_{\rho / 2}\left(\mathbf{x}_{0}\right)}\left[|\nabla Q|^{2}+f_{b}(Q)\right] d \mathbf{x} \leq & \mu_{2} \int_{B_{2 \rho\left(\mathbf{x}_{0}\right) \backslash B_{\rho / 2}\left(\mathbf{x}_{0}\right)}}\left[|\nabla Q|^{2}+f_{b}(Q)\right] d \mathbf{x} \\
& +C_{7} \rho^{2}
\end{aligned}
$$

where $\mu_{1}, \mu_{2}$, and $C_{7}$ are positive constants depending only on the constants in (1.2) and (1.4). The above inequality allows us to do a "hole filling" argument. Adding $\mu_{2} \int_{B_{\rho / 2}}\left[|\nabla Q|^{2}+\right.$ $\left.f_{b}(Q)\right] d \mathbf{x}$ to both sides we obtain

$$
\begin{aligned}
\int_{B_{\rho / 2}\left(\mathbf{x}_{0}\right)}\left[|\nabla Q|^{2}+f_{b}(Q)\right] d \mathbf{x} \leq & \theta \int_{B_{2 \rho}\left(\mathbf{x}_{0}\right)}\left[|\nabla Q|^{2}+f_{b}(Q)\right] d \mathbf{x} \\
& +C_{8} \rho^{2}
\end{aligned}
$$


where $\theta=\frac{\mu_{2}}{\mu_{1}+\mu_{2}}<1$ for all $\mathbf{x} \in B_{r}(\mathbf{o})$ and $\rho \leq r \leq r_{0}$. Let $\gamma>0$ satisfy $\theta=\left(\frac{1}{4}\right)^{\gamma}$ and set

$$
w_{\mathbf{x}_{0}}(\rho)=\int_{B_{\rho}\left(\mathbf{x}_{0}\right)}\left[|\nabla Q|^{2}+f_{b}(Q)\right] d \mathbf{x},
$$

then

$$
w_{\mathbf{x}_{0}}\left(\frac{\rho}{4}\right) \leq\left(\frac{1}{4}\right)^{\gamma} w_{\mathbf{x}_{0}}(\rho)+C_{8} \rho^{2} .
$$

We obtain, just as in the proof of Lemma 2.1 from [6], Ch. III the Morrey-type estimate

$$
w_{\mathbf{x}_{0}}(\rho) \leq C\left(\frac{\rho}{r}\right)^{2 \sigma}\left[w_{\mathbf{x}_{0}}(r)+r^{2 \sigma}\right]
$$

for all $\mathbf{x}_{0} \in B_{r}(\mathbf{o})$ and $\rho \leq \frac{r}{2} \leq \frac{r_{0}}{2}$ where $\sigma>0$ depends on $\theta$, and $C$ depends on $\theta, r_{0}$, and $C_{8}$. Since $Q$ is bounded, the theorem follows from this and Morrey's theorem. (See [6], Ch. III, Theorems 1.1 and 1.2.)

Proof of Theorem 2 Since $\partial \Omega$ is of class $C^{2}$ there exists $0<\rho_{0} \leq r_{1}$ so that $\Omega$ satisfies the exterior sphere condition at each $\mathbf{y} \in \partial \Omega$ with a ball $B_{\rho_{0}}$ of radius $\rho_{0}$. It follows that for each $\mathbf{x} \in \Omega$ and $s>0, B_{s}(\mathbf{x}) \cap \Omega$ also satisfies the exterior sphere condition at each $\mathbf{y} \in \partial\left(B_{S}(\mathbf{x}) \cap \Omega\right)$ with a ball $B_{\rho_{0}}$. Assume the hypotheses of Theorem 2. Let $\mathbf{o} \in \partial \Omega$, $\mathbf{x}_{0} \in B_{r}(\mathbf{o}) \cap \Omega, 0<\rho \leq r, \frac{\rho}{2} \leq s \leq \rho$, and using Definition 2.1 set

$$
Q_{s}(\mathbf{x}):=Q_{B_{s}\left(\mathbf{x}_{0}\right)}(\mathbf{x}) .
$$

Just as in the Proof of Theorem 1, we have

$$
\begin{aligned}
& \int_{B_{\rho / 2}\left(\mathbf{x}_{0}\right) \cap \Omega}\left[\alpha_{1}|\nabla Q|^{2}+f_{b}(Q)\right] d \mathbf{x} \leq \mathcal{F}\left(Q ; B_{2 \rho}\left(\mathbf{x}_{0}\right) \cap \Omega\right)+C_{0} \rho^{2} \\
& \leq \mathcal{F}\left(Q_{s} ; B_{2 \rho}\left(\mathbf{x}_{0}\right) \cap \Omega\right)+C_{0} \rho^{2} \\
& \leq \int_{B_{s}\left(\mathbf{x}_{0}\right) \cap \Omega}\left[\alpha_{2}|\nabla H|^{2}+f_{b}(H)\right] d \mathbf{x}+\mathcal{F}\left(Q ;\left(B_{2 \rho}\left(\mathbf{x}_{0}\right) \cap \Omega\right) \backslash B_{S}\left(\mathbf{x}_{0}\right)\right) \\
& \quad+C_{1} \rho^{2} .
\end{aligned}
$$

Assume for now that $H$ is separated from $\partial \mathcal{M}$ on $\partial\left(B_{s}\left(\mathbf{x}_{0}\right) \cap \Omega\right)$. Then $f$ is $C^{\infty}$ on a neighborhood of $\left.H\right|_{B_{s}\left(\mathbf{x}_{0}\right) \cap \Omega}$ and

$$
\Delta f(H) \geq 0 \text { on } B_{s}\left(\mathbf{x}_{0}\right) \cap \Omega .
$$

We next solve

$$
\begin{array}{rll}
\Delta w=1 & \text { in } & B_{S}\left(\mathbf{x}_{0}\right) \cap \Omega, \\
w=0 & \text { on } & \partial\left(B_{s}\left(\mathbf{x}_{0}\right) \cap \Omega\right)
\end{array}
$$

and note that since $B_{S}\left(\mathbf{x}_{0}\right) \cap \Omega$ satisfies the exterior sphere condition at each boundary point as described above we can construct a barrier function relative to each point in $\partial\left(B_{S}\left(\mathbf{x}_{0}\right) \cap \Omega\right)$ so as to ensure that the normal derivative

$$
\left|\partial_{\nu} w(\mathbf{y})\right| \leq C_{2} s \text { for each } \mathbf{y} \in \partial\left(B_{s}\left(\mathbf{x}_{0}\right) \cap \Omega\right) \text { for which } \partial_{\nu} w(\mathbf{y}) \text { exists. }
$$

To see this let $B_{\rho_{0}}(\mathbf{z})$ be the exterior sphere associated with $\mathbf{y}$ (so that $\bar{B}_{\rho_{0}}(\mathbf{z}) \bigcap\left(\overline{B_{s}\left(\mathbf{x}_{0}\right) \cap \Omega}\right)$ $=\{\mathbf{y}\})$ and set $\eta=\eta(\mathbf{x})=|\mathbf{x}-\mathbf{z}|$. Here we can take $\rho_{0}=\rho_{0}(\partial \Omega)>0$ fixed and assume 
without loss of generality that $s<\rho_{0}$. Then $B_{s}\left(\mathbf{x}_{0}\right) \cap \Omega \subset B_{\left(2 s+\rho_{0}\right)}(\mathbf{z}) \backslash B_{\rho_{0}}(\mathbf{z})$ and the function

$$
g(\eta(\mathbf{x})) \equiv g(\eta)=\rho_{0}^{2}\left(3+\frac{6 s}{\rho_{0}}\right) \ln \left(\frac{\rho_{0}}{\eta}\right)+3 \rho_{0}\left(\eta-\rho_{0}\right)
$$

satisfies

$$
g\left(\rho_{0}\right)=0, g(\eta) \leq 0 \text { and } \Delta g \geq 1 \text { for } \rho_{0} \leq \eta \leq \rho_{0}+2 s .
$$

This can then be used as a barrier so that $\left|\partial_{v} w(\mathbf{y})\right| \leq\left|g^{\prime}\left(\rho_{0}\right)\right|=6 s$. The set $B_{s}\left(\mathbf{x}_{0}\right) \cap \Omega$ is such that $w \in H^{2}\left(B_{S}\left(\mathbf{x}_{0}\right) \cap \Omega\right)$ and we have

$$
\begin{gathered}
\int_{B_{s}\left(\mathbf{x}_{0}\right) \cap \Omega} f(H) d \mathbf{x}=\int_{B_{S}\left(\mathbf{x}_{0}\right) \cap \Omega} w \Delta f(H) d \mathbf{x}+\int_{\partial\left(B_{S}\left(\mathbf{x}_{0}\right) \cap \Omega\right)} f(Q) \partial_{\nu} w d \mathbf{s} \\
\leq \int_{\partial\left(B_{s}\left(\mathbf{x}_{0}\right) \cap \Omega\right)} f(Q) \partial_{\nu} w d \mathbf{s} .
\end{gathered}
$$

It follows that

$$
\int_{B_{s}\left(\mathbf{x}_{0}\right) \cap \Omega} f_{b}(H) d \mathbf{x} \leq C_{3} s \int_{\partial\left(B_{s}\left(\mathbf{x}_{0}\right) \cap \Omega\right)} f_{b}(Q) d \mathbf{s}+C_{4} s^{2} .
$$

We remark that one needs $H(\mathbf{x})$ and $f(H(\mathbf{x}))$ in $H^{2}\left(B_{S}\left(\mathbf{x}_{0}\right) \cap \Omega\right)$ to directly apply Green's identity as above, however these functions are only in $H^{1}\left(B_{S}\left(\mathbf{x}_{0}\right) \cap \Omega\right)$. We can circumvent this problem by using an approximation. We assume without loss of generality that $s$ is sufficiently small so that $B_{s}\left(\mathbf{x}_{0}\right) \cap \Omega$ is strictly star-like with respect to a point $\mathbf{p} \in B_{s}\left(\mathbf{x}_{0}\right) \cap \Omega$ and set $H_{\lambda}(\mathbf{x})=H(\mathbf{p}+\lambda(\mathbf{x}-\mathbf{p}))$. Then for $0<\lambda<1$ we have $H_{\lambda}$ and $f\left(H_{\lambda}\right)$ in $C^{\infty}\left(\overline{\left(B_{S}\left(\mathbf{x}_{0}\right) \cap \Omega\right)}\right)$, such that $H_{\lambda}$ is harmonic, $f\left(H_{\lambda}\right)$ is subharmonic, and such that $H_{\lambda} \rightarrow H, f\left(H_{\lambda}\right) \rightarrow f(H)$ in $H^{1}\left(B_{S}\left(\mathbf{x}_{0}\right) \cap \Omega\right)$ as $\lambda \uparrow 1$. We can then apply Green's identity for $\lambda<1$ and obtain (2.7) in the limit as $\lambda \uparrow 1$.

We next replace the condition that $H$ is separated from $\partial \mathcal{M}$ on $\partial\left(B_{S}\left(\mathbf{x}_{0}\right) \cap \Omega\right)$ with the condition that $\int_{\partial\left(B_{s}\left(\mathbf{x}_{0}\right) \cap \Omega\right)} f_{b}(Q) d \mathbf{s}<\infty$. This is done just as before by considering $\tau H$ for $0<\tau<1$ for which (2.7) is valid and letting $\tau \uparrow 1$.

Combining (2.6) and (2.7) we have

$$
\begin{aligned}
\int_{B_{\rho / 2}\left(\mathbf{x}_{0}\right) \cap \Omega}\left[\alpha_{1}|\nabla Q|^{2}+f_{b}(Q)\right] d \mathbf{x} \leq & \alpha_{2} \int_{B_{s}\left(\mathbf{x}_{0}\right) \cap \Omega}|\nabla H|^{2} d \mathbf{x} \\
& +C_{5} s \int_{\partial\left(B_{s}\left(\mathbf{x}_{0}\right) \cap \Omega\right)} f_{b}(Q) d \mathbf{s} \\
& +\mathcal{F}\left(Q ;\left(B_{2 \rho}\left(\mathbf{x}_{0}\right) \backslash B_{s}\left(\mathbf{x}_{0}\right)\right) \cap \Omega\right)+C_{6} \rho^{2} .
\end{aligned}
$$

We write $\partial\left(B_{S}\left(\mathbf{x}_{0}\right) \cap \Omega\right)=\left(\partial B_{S}\left(\mathbf{x}_{0}\right) \cap \Omega\right) \cup\left(B_{S}\left(\mathbf{x}_{0}\right) \cap \partial \Omega\right)$. Then using (1.5) we have

$$
\int_{\partial\left(B_{s}\left(\mathbf{x}_{0}\right) \cap \Omega\right)} f_{b}(Q) d \mathbf{s} \leq \int_{\partial B_{s}\left(\mathbf{x}_{0}\right) \cap \Omega} f_{b}(Q) d \mathbf{s}+C_{7}
$$

and it follows that

$$
\begin{aligned}
\int_{B_{\rho / 2}\left(\mathbf{x}_{0}\right) \cap \Omega}\left[\alpha_{1}|\nabla Q|^{2}+f_{b}(Q)\right] d \mathbf{x} \leq & \alpha_{2} \int_{B_{s}\left(\mathbf{x}_{0}\right) \cap \Omega}|\nabla H|^{2} d \mathbf{x} \\
& +C_{5} s \int_{\partial B_{s}\left(\mathbf{x}_{0}\right) \cap \Omega} f_{b}(Q) d \mathbf{s} \\
& +\mathcal{F}\left(Q ;\left(B_{2 \rho}\left(\mathbf{x}_{0}\right) \backslash B_{s}\left(\mathbf{x}_{0}\right)\right) \cap \Omega\right)+C_{8} \rho .
\end{aligned}
$$


At this point we argue just as in the Proof of Theorem 1 arriving at

$$
\begin{aligned}
\beta_{1} \int_{B_{\rho / 2}\left(\mathbf{x}_{0}\right) \cap \Omega}\left[|\nabla Q|^{2}+f_{b}(Q)\right] d \mathbf{x} \leq & \beta_{2} \int_{\left(B_{2 \rho}\left(\mathbf{x}_{0}\right) \cap \Omega\right) \backslash B_{\rho / 2}\left(\mathbf{x}_{0}\right)}\left[|\nabla Q|^{2}+f_{b}(Q)\right] d \mathbf{x} \\
& +C_{9} \rho
\end{aligned}
$$

for fixed positive constants $\beta_{1}, \beta_{2}$, and $C_{9}$, where here they depend on $Q_{0}$ as well. We next extend $Q$ in a neighborhood of $\Omega^{\prime}$ of $\bar{\Omega}$ so that

$$
Q \in H^{1}\left(\Omega^{\prime} ; \overline{\mathcal{M}}\right) \cap C^{0,1}\left(\Omega^{\prime} \backslash \Omega ; \overline{\mathcal{M}}\right)
$$

and extend $f_{b} \equiv 0$ in $\Omega^{\prime} \backslash \bar{\Omega}$. We then have

$$
\begin{aligned}
\beta_{1} \int_{B_{\rho / 2}\left(\mathbf{x}_{0}\right)}\left[|\nabla Q|^{2}+f_{b}\right] d \mathbf{x} \leq & \beta_{2} \int_{B_{2 \rho}\left(\mathbf{x}_{0}\right) \backslash B_{\rho / 2}\left(\mathbf{x}_{0}\right)}\left[|\nabla Q|^{2}+f_{b}\right] d \mathbf{x} \\
& +C_{10} \rho
\end{aligned}
$$

for all $\mathbf{x}_{0}$ in a neighborhood of $\partial \Omega$ and all $\rho$ sufficiently small. Our assertion follows just as in the proof for Theorem 1.

Proof of Corollary 2 To prove the interior regularity result, let $B$ be an open disk in $\Omega$ and $Q$ a local minimizer for $\mathcal{F}$ in $H^{1}(B ; \overline{\mathcal{M}})$. Assume $\overline{B_{4 r}(\mathbf{o})} \subset B \backslash \Lambda(Q)$. As outlined in Sect. 1 it is enough to consider solutions to the quasilinear Euler-Lagrange equation for $\mathcal{F}^{\prime}$ from (1.7) satisfying the strong ellipticity condition (1.8) in $\overline{B_{4 r}(\mathbf{o})}$. It suffices to show that our solutions have Hölder-continuous first derivatives in $B_{r}(\mathbf{o})$; higher regularity then follows using techniques from linear theory [6,10]. Note that $Q=\tilde{Q}(\mathbf{z}(\mathbf{x})), f_{b}(\tilde{Q}(\mathbf{z}))$ is a $C^{\infty}$ function if $\mathbf{z}$ in a neighborhood of the range of $\mathbf{z}(\mathbf{x})$ for $\mathbf{x}$ in $\overline{B_{4 r}(\mathbf{o})}$, and the coefficients of $f_{l d}$ are polynomials in $\mathbf{z}$. By Theorem $1, \mathbf{z}(\mathbf{x}) \in C^{\sigma}\left(\overline{B_{4 r}(\mathbf{o})}\right)$ and we can apply the result from [6], $\mathrm{Ch}$. VI, Proposition1 asserting that in two space dimensions a continuous weak solution is in $H^{2, p}$ for some $p>2$, and thus, first order derivations are Hölder continuous. The argument given there proves an interior estimate. To prove regularity near the boundary, we assume $Q$ is a local minimizer in $\Omega \cap B_{4 r}(\mathbf{o})$, where $\mathbf{o} \in \partial \Omega$ and $r<r_{1}, B_{4 r}(\mathbf{o}) \cap \partial \Omega$ is of class $C^{k, \alpha}$ for some $k \geq 2$ and $\alpha \in(0,1), Q_{0} \in C^{k, \alpha}\left(B_{4 r}(\mathbf{o}) \cap \partial \Omega\right)$, and $\overline{B_{4 r}(\mathbf{o})} \cap \Lambda(Q)=\emptyset$. As before, $Q=\tilde{Q}(\mathbf{z}(\mathbf{x}))$ and $f_{p}(\tilde{Q}(\mathbf{z}))$ is a $C^{\infty}$ function if $\mathbf{z}$ on a neighborhood of the range of $\mathbf{z}(\mathbf{x})$ for $\mathbf{x} \in \overline{B_{4 r}(\mathbf{o}) \cap \Omega}$. Here it is enough to prove that $Q$ has Hölder-continuous first derivatives in $B_{\frac{r}{2}}(\mathbf{o}) \cap \bar{\Omega}$. One can derive a reverse-Hölder type inequality on sets $B_{2 r}\left(\mathbf{x}_{0}\right) \cap \Omega$ where $\mathbf{x}_{0} \in B_{2 r}(\mathbf{o}) \cap \partial \Omega$ in the spirit of that given in [6], Ch. V, Sec. 2, and then apply the same argument to prove the estimate near the boundary asserting that the solution is in $H^{2, p}\left(B_{\frac{r}{2}}(\mathbf{o}) \cap \Omega\right)$. The details for this inequality are given in the Appendix. As before, higher regularities follows from linear theory.

\section{Analysis of $\Lambda(Q)$}

In Sect. 2 we proved that a local minimizer on a ball $B_{4 \bar{r}}(\mathbf{o}) \subset \Omega$ satisfies

$$
\|Q\|_{C^{\delta}\left(\overline{B_{\bar{r}}(\mathbf{o})}\right)} \leq A_{1}
$$

for a fixed $0<\delta<1$ by establishing the Morrey-type estimate

$$
\int_{B_{\rho}(\mathbf{y})}\left[|\nabla Q|^{2}+f_{b}(Q)\right] d \mathbf{x} \leq A_{2} \rho^{2 \delta}
$$


for all $\mathbf{y} \in B_{\bar{r}}(\mathbf{o})$ and $0<\rho \leq \bar{r}$. This was done by constructing comparison functions using harmonic replacements for each component of $Q$. In this section we consider $f_{e}$ satisfying (1.9) and (1.10), and prove that if $Q$ is a finite energy local minimizer for $\mathcal{F}$ on an open set $E$ then $\Lambda(Q) \cap E=\emptyset$. We do this by refining the notion of replacement in this case so as to prove that (3.2) holds for any $0<\delta<1$. We are then able to carry this one step further to get

$$
\int_{B_{\rho}(\mathbf{o})} f_{b}(Q) d \mathbf{x} \leq A_{3} \rho^{2}
$$

for all $0<\rho$ sufficiently small. If $\mathbf{o} \in \Lambda(Q)$ we know that $\lim _{\mathbf{y} \rightarrow \mathbf{0}} f_{b}(Q(\mathbf{y}))=\infty$ and if $\Lambda(Q) \cap E$ is nonempty, this leads to a contradiction.

Proof of Theorem 3 Assume $f_{e}$ satisfies (1.9) and (1.10) and let $Q$ be a local minimizer for $\mathcal{F}$ on $B_{4 \bar{r}}(\mathbf{o}) \subset \Omega$. Assume without loss of generality that $\mathbf{o} \in \Lambda(Q)$. We associate with $f_{e}$ and any $V \in \overline{\mathcal{M}}$ the quadratic form corresponding to

$$
L(V)[\nabla g]=\sum_{i, j=1}^{2}\left[L_{1} \delta_{i j}+L_{5} V_{i j}\right] g_{x_{i}} g_{x_{j}} .
$$

Using (1.10) we have that there exist constants $\mu_{1}, \mu_{2}>0$ so that

$$
\begin{gathered}
\mu_{1}|\zeta|^{2} \leq L(V)[\zeta] \leq \mu_{2}|\zeta|^{2} \text { for all } \zeta \in \mathbb{R}^{2} \\
\text { and } V \in \overline{\mathcal{M}} .
\end{gathered}
$$

Let $\mathbf{y}=A \mathbf{x}$ be a linear nonsingular change of variables in $\mathbb{R}^{2}$. Given $V(\mathbf{x}) \in \overline{\mathcal{M}}$ set $\hat{V}(\mathbf{y})=V\left(A^{-1}(\mathbf{y})\right)$ and define the quadratic form

$$
\hat{L}(\hat{V}(\mathbf{y}))[\zeta]:=L(V(\mathbf{x}))\left[A^{T} \zeta\right]=\sum_{i, j=1}^{2} c_{i j}(\hat{V}(\mathbf{y})) \zeta_{i} \zeta_{j} .
$$

Here

$$
c_{i j}(\hat{V}(\mathbf{y}))=\left[L_{1} A A^{t}+A \hat{W}(\mathbf{y}) A^{t}\right]_{i j} \text { for } 1 \leq i, j \leq 2
$$

where $\hat{W}(\mathbf{y})$ is the $2 \times 2$ matrix whose $l k$ entry is the $l k$ entry of $\hat{V}(\mathbf{y})$. Thus $c_{i j}(\hat{V}(\mathbf{y}))$ are affine functions of $\hat{V}_{l k}(\mathbf{y})$ for $1 \leq l, k \leq 2$. Choose and fix $A$ so that $c_{i j}(\hat{Q}(\mathbf{o}))=c_{i j}(Q(\mathbf{o}))=\delta_{i j}$. Note that $c_{i j}=c_{j i}$ and $\tilde{\mu}_{1}|\zeta|^{2} \leq c_{i j} \zeta_{i} \zeta_{j} \leq \tilde{\mu}_{2}|\zeta|^{2}$ where $0<\tilde{\mu}_{1} \leq \tilde{\mu}_{2}$ are constants independent of $\hat{V}(\mathbf{y})$ for $\hat{V} \in \overline{\mathcal{M}}$. Set $\mathcal{E}=A\left[B_{\bar{r}}(\mathbf{o})\right]$ and let $r \leq \bar{R}$ where $\bar{R}$ satisfies $B_{4 \bar{R}}(\mathbf{o}) \subset \mathcal{E}$. By the chain rule, the energy $\mathcal{F}$ transforms to

$$
\hat{F}(\hat{V} ; \mathcal{E})=\int_{\mathcal{E}}\left[\hat{f_{e}}(\hat{V}, \nabla \hat{V})+f_{b}(\hat{V})\right]\left|\operatorname{det} A^{-1}\right| d \mathbf{y}
$$

where

$$
\hat{f}_{e}(\hat{V}, \nabla \hat{V})=\sum_{l, k=1}^{3} \hat{L}(\hat{V})\left[\nabla \hat{V}_{l k}\right]+\mathbf{b} \cdot \nabla \hat{V}
$$

such that

$$
\mathbf{b}=\left[b_{l k m}(\hat{V})\right], \quad b_{l k m}(\hat{V})=\sum_{i, j=1}^{2} b_{l k m}^{i j} \hat{V}_{i j}
$$


and $\left\{b_{l k m}^{i j}\right\}$ are all constants that are uniformly bounded independent of $\hat{V}$ for $\hat{V} \in \mathcal{M}$. For $\overline{\mathbf{y}} \in B_{r}(\mathbf{o})$ let $\mathcal{L}(\overline{\mathbf{y}})$ be the constant coefficient scalar elliptic operator

$$
\mathcal{L}(\overline{\mathbf{y}})[\hat{g}]=\sum_{i, j=1}^{2} c_{i j}(\hat{Q}(\overline{\mathbf{y}})) \hat{g}_{y_{i} y_{j}} .
$$

Let $0<s \leq r$. Then $B_{S}(\overline{\mathbf{y}}) \subset \mathcal{E}$ and we can construct the $\mathcal{L}$-replacement comparison function $\hat{Q}_{s}(\mathbf{y}):=\hat{Q}_{B_{s}(\overline{\mathbf{y}})}(\mathbf{y})$ such that for each $1 \leq \ell, k \leq 3$

$$
\begin{array}{cc}
\mathcal{L}(\overline{\mathbf{y}})\left[\hat{Q}_{s l k}\right]=0 & \text { on } B_{s}(\overline{\mathbf{y}}), \\
\hat{Q}_{s l k}=\hat{Q}_{l k} & \text { on } \partial B_{s}(\overline{\mathbf{y}}), \\
\hat{Q}_{s l k}=\hat{Q}_{l k} & \text { on } \mathcal{E} \backslash B_{s}(\overline{\mathbf{y}}) .
\end{array}
$$

In the following estimates the constants $M_{i}$ depend on $A_{2}$ and $\delta$ from (3.2), however they are independent of $s$ and $r$ for $0<s \leq r \leq r_{0}$. Since homogeneous solutions to $\mathcal{L}(\overline{\mathbf{y}})$ satisfy the strong maximum principle $\hat{Q}_{s}$ has the same properties as the harmonic replacements used earlier. We use two other features of $\hat{Q}_{s}$. Since $\hat{Q}_{s l k}$ minimizes

$$
\int_{B_{s}(\overline{\mathbf{y}})} c_{i j}(\hat{Q}(\overline{\mathbf{y}})) g_{y_{i}} g_{y_{j}} d \mathbf{y}
$$

among $g \in H^{1}\left(B_{S}(\overline{\mathbf{y}})\right)$ satisfying $g=\hat{Q}_{l k}$ on $\partial B_{S}(\overline{\mathbf{y}})$ it follows that

$$
\int_{B_{s}(\overline{\mathbf{y}})}\left|\nabla \hat{Q}_{s}\right|^{2} d \mathbf{y} \leq M_{1} \int_{B_{s}(\overline{\mathbf{y}})}|\nabla \hat{Q}|^{2} d \mathbf{y}
$$

where $M_{1}=M_{1}\left(\tilde{\mu}_{1}, \tilde{\mu}_{2}\right)$. Second, from the maximum principle and (3.1) we have

$$
\underset{B_{s}(\overline{\mathbf{y}})}{\operatorname{osc}} \hat{Q}_{s_{i j}} \leq \underset{\partial B_{s}(\overline{\mathbf{y}})}{\operatorname{osc}} \hat{Q}_{s_{i j}}=\underset{\partial B_{s}(\overline{\mathbf{y}})}{\operatorname{osc}} \hat{Q}_{i j} \leq M_{2} s^{\delta} \text { for each } i \text { and } j .
$$

Just as before if $\left.\hat{Q}_{S}\right|_{\partial B_{s}(\overline{\mathbf{y}})}$ is separated from $\partial \mathcal{M}$ then the function $f\left(\hat{Q}_{s}(\overline{\mathbf{y}})\right)$ is a classical $\mathcal{L}$-subsolution on $B_{S}(\overline{\mathbf{y}})$. Set

$$
u(\mathbf{y})=\frac{1}{4}\left(|\mathbf{y}-\overline{\mathbf{y}}|^{2}-s^{2}\right)
$$

and let $w(y)$ solve

$$
\begin{aligned}
\mathcal{L}(\overline{\mathbf{y}})[w]=1 & \text { on } B_{s}(\overline{\mathbf{y}}), \\
w=0 & \text { on } \partial B_{S}(\overline{\mathbf{y}}) .
\end{aligned}
$$

Then

$$
\begin{aligned}
\mathcal{L}(\overline{\mathbf{y}})[w-u] & =1-\frac{1}{2} \sum_{i=1}^{2} c_{i i}(\hat{Q}(\overline{\mathbf{y}})) \\
& =\frac{1}{2} \sum_{i=1}^{2}\left(c_{i i}(\hat{Q}(\mathbf{o}))-c_{i i}(\hat{Q}(\overline{\mathbf{y}}))=C \text { on } B_{s}(\overline{\mathbf{y}})\right.
\end{aligned}
$$

where $C$ is constant on $B_{S}(\overline{\mathbf{y}})$ such that

$$
|C| \leq M_{3}|\hat{Q}(\mathbf{o})-\hat{Q}(\overline{\mathbf{y}})| \leq M_{4} r^{\delta} .
$$


Here we have used (3.1) and the formulas for the $c_{i j}$. It follows that

$$
|\nabla w-\nabla u| \leq M_{5} s r^{\delta} \quad \text { on } \quad \overline{B_{s}}(\overline{\mathbf{y}}) .
$$

In particular

$$
\left|\nabla w(\mathbf{y})-\frac{s}{2} v\right| \leq M_{6} s r^{\delta} \quad \text { for } \mathbf{y} \in \partial B_{S}(\overline{\mathbf{y}}) \quad \text { and } \quad v=\frac{(\mathbf{y}-\overline{\mathbf{y}})}{s} .
$$

Also, since $f\left(\hat{Q}_{S}\right)$ is a subsolution for $\mathcal{L}(\overline{\mathbf{y}})$, we have

$$
\begin{aligned}
\int_{B_{S}(\overline{\mathbf{y}})} f\left(\hat{Q}_{s}\right) d \mathbf{y} & =\int_{B_{S}(\overline{\mathbf{y}})} f\left(\hat{Q}_{s}\right) \mathcal{L}(\overline{\mathbf{y}})[w] d \mathbf{y} \\
& \leq \int_{\partial B_{s}(\overline{\mathbf{y}})} f\left(\hat{Q}_{s}\right) \nu_{\ell} c_{l k}(\hat{Q}(\overline{\mathbf{y}})) w_{y_{k}} d \mathbf{s} .
\end{aligned}
$$

We need to express this in terms of $f_{b}$. In order to do this set

$$
\mathfrak{I}=\int_{\partial B_{s}(\overline{\mathbf{y}})}\left(-\kappa\left|\hat{Q}_{s}\right|^{2}+b_{0}\right) v_{\ell} c_{l k}(\hat{Q}(\overline{\mathbf{y}})) w_{y_{k}} d \mathbf{s} .
$$

Since $c_{l k}(Q)$ is a fixed affine function such that $c_{l k}(Q(\mathbf{o}))=\delta_{l k}$ it follows that

$$
\left|v_{\ell} c_{l k}(\hat{Q}(\overline{\mathbf{y}})) w_{y_{k}}-\frac{s}{2}\right| \leq M_{7} s r^{\delta} .
$$

Adding $\mathfrak{I}$ to the right side of (3.7) then gives

$$
\int_{\partial B_{s}(\overline{\mathbf{y}})} f_{b}\left(\hat{Q}_{s}\right) \nu_{\ell} c_{l k}(\hat{Q}(\overline{\mathbf{y}})) w_{y_{k}} d \mathbf{s} \leq s\left(\frac{1}{2}+M_{8} r^{\delta}\right) \int_{\partial B_{s}(\overline{\mathbf{y}})} f_{b}\left(\hat{Q}_{s}\right) d \mathbf{s} .
$$

Also from (3.8) we get

$$
\left|\mathfrak{I}-\frac{s}{2} \int_{\partial B_{s}}\left(-\kappa\left|\hat{Q}_{s}\right|^{2}+b_{0}\right) d \mathbf{s}\right| \leq M_{9} s^{2} r^{\delta} .
$$

Next, using the fact that $\int_{B_{S}} c d \mathbf{y}=\frac{s}{2} \int_{\partial B_{s}} c d \mathbf{s}$ if $c$ is a constant and using (3.6) we have the estimate

$$
\left|\mathfrak{I}-\int_{B_{s}(\overline{\mathbf{y}})}\left(-\kappa\left|\hat{Q}_{s}\right|^{2}+b_{0}\right) d \mathbf{y}\right| \leq M_{10}\left(s^{2+\delta}+s^{2} r^{\delta}\right) \leq M_{11} s^{2} r^{\delta} .
$$

Thus adding $\mathfrak{I}$ to the left side of (3.7) together with (3.9) gives

$$
\int_{B_{s}(\overline{\mathbf{y}})} f_{b}\left(\hat{Q}_{s}\right) d \mathbf{y} \leq s\left(\frac{1}{2}+M_{8} r^{\delta}\right) \int_{\partial B_{s}(\overline{\mathbf{y}})} f_{b}(\hat{Q}) d \mathbf{s}+M_{12} s^{2} r^{\delta} .
$$

Just as before we can drop the assumption that $\left.\hat{Q}\right|_{\partial B_{s}(\overline{\mathbf{y}})}$ is separated from $\partial \mathcal{M}$ and replace it with the condition that $f_{b}(\hat{Q}) \in L^{1}\left(\partial B_{s}(\overline{\mathbf{y}})\right)$. Also as in Sect. 2, using $\hat{Q}_{s}$ as a comparison function and the above inequality we get

$$
\begin{aligned}
\int_{B_{s}(\overline{\mathbf{y}})}\left[\hat{f}_{e}(\hat{Q}, \nabla \hat{Q})+f_{b}(\hat{Q})\right] d \mathbf{y} \leq & \int_{B_{s}(\overline{\mathbf{y}})} \hat{f}_{e}\left(\hat{Q}_{s}, \nabla \hat{Q}_{s}\right) d \mathbf{y} \\
& +s\left(\frac{1}{2}+M_{8} r^{\delta}\right) \int_{\partial B_{s}(\overline{\mathbf{y}})} f_{b}(\hat{Q}) d \mathbf{s} \\
& +M_{12} s^{2} r^{\delta} .
\end{aligned}
$$


We rewrite this as

$$
\begin{aligned}
& \int_{B_{s}(\overline{\mathbf{y}})}\left[\hat{f}_{e}(\hat{Q}(\overline{\mathbf{y}}), \nabla \hat{Q})-\hat{f}_{e}\left(\hat{Q}(\overline{\mathbf{y}}), \nabla \hat{Q}_{s}\right)\right] d \mathbf{y} \\
& \leq \quad \int_{B_{s}(\overline{\mathbf{y}})}\left[\left(\hat{f}_{e}(\hat{Q}(\overline{\mathbf{y}}), \nabla \hat{Q})-\hat{f}_{e}(\hat{Q}, \nabla \hat{Q})\right)\right. \\
& \left.\quad+\left(\hat{f}_{e}\left(\hat{Q}_{s}, \nabla \hat{Q}_{s}\right)-\hat{f}_{e}\left(\hat{Q}(\overline{\mathbf{y}}), \nabla \hat{Q}_{s}\right)\right)\right] d \mathbf{y} \\
& \quad-\int_{B_{s}(\overline{\mathbf{y}})} f_{b}(\hat{Q}) d \mathbf{y}+s\left(\frac{1}{2}+M_{8} r^{\delta}\right) \int_{\partial B_{s}(\overline{\mathbf{y}})} f_{b}(\hat{Q}) d \mathbf{s}+M_{12} s^{2} r^{\delta}
\end{aligned}
$$

The left side of (3.10) equals

$$
\begin{aligned}
& \int_{B_{s}(\overline{\mathbf{y}})} c_{i j}\left(\hat{Q}(\overline{\mathbf{y}})\left[\hat{Q}_{l k, y_{i}} \hat{Q}_{l k, y_{j}}-\hat{Q}_{s l k, y_{i}} \hat{Q}_{s l k, y_{j}}\right] d \mathbf{y}\right. \\
& \quad+\int_{B_{s}(\overline{\mathbf{y}})} \mathbf{b}(\hat{Q}(\overline{\mathbf{y}})) \cdot \nabla\left(\hat{Q}-\hat{Q}_{s}\right) d \mathbf{y} .
\end{aligned}
$$

Using (3.3) and (3.4) the first integral in (3.11) equals and satisfies

$$
\begin{aligned}
& \int_{B_{s}(\overline{\mathbf{y}})} c_{i j}(\hat{Q}(\overline{\mathbf{y}}))\left(\hat{Q}_{l k, y_{i}}-\hat{Q}_{s l k, y_{i}}\right)\left(\hat{Q}_{l k, y_{j}}+\hat{Q}_{s l k, y_{j}}\right) d \mathbf{y} \\
& \quad=\int_{B_{s}(\overline{\mathbf{y}})} c_{i j}(\hat{Q}(\overline{\mathbf{y}}))\left(\hat{Q}_{l k, y_{i}}-\hat{Q}_{s l k, y_{i}}\right)\left(\hat{Q}_{l k, y_{j}}-\hat{Q}_{s l k, y_{j}}\right) d \mathbf{y} \\
& \quad \geq \tilde{\mu}_{1} \int_{B_{s}(\overline{\mathbf{y}})}\left|\nabla\left(\hat{Q}-\hat{Q}_{s}\right)\right|^{2} d \mathbf{y} .
\end{aligned}
$$

For the second integral in (3.11), note that $\mathbf{b}(\hat{Q}(\overline{\mathbf{y}}))$ is constant and $\hat{Q}=\hat{Q}_{s}$ on $\partial B_{s}(\overline{\mathbf{y}})$. Thus this integral is zero.

The first integral on the right side of (3.10) can be estimated using (3.1), (3.2), (3.5), (3.6) and the formula for $\hat{f}_{e}$. We find that it is bounded by

$$
M_{13}\left(s^{\delta} \int_{B_{s}(\overline{\mathbf{y}})}\left[|\nabla \hat{Q}|^{2}+\left|\nabla \hat{Q}_{s}\right|^{2}\right] d \mathbf{y}+s^{1+\delta}\left(\int_{B_{s}(\overline{\mathbf{y}})}\left[|\nabla \hat{Q}|^{2}+\left|\nabla \hat{Q}_{s}\right|^{2}\right] d \mathbf{y}\right)^{1 / 2}\right) \leq M_{14} s^{3 \delta} .
$$

We arrive at

$$
\begin{aligned}
\tilde{\mu}_{1} \int_{B_{s}(\overline{\mathbf{y}})}\left|\nabla\left(\hat{Q}-\hat{Q}_{s}\right)\right|^{2} d \mathbf{y} \leq & M_{15}\left(s^{3 \delta}+s^{2} r^{\delta}\right) \\
& -\int_{B_{s}(\overline{\mathbf{y}})} f_{b}(\hat{Q}) d \mathbf{y}+s\left(\frac{1}{2}+M_{8} r^{\delta}\right) \int_{\partial B_{s}(\overline{\mathbf{y}})} f_{b}(\hat{Q}) d \mathbf{s} .
\end{aligned}
$$

Thus we have

$$
\begin{aligned}
\tilde{\mu}_{1} \int_{B_{s}(\overline{\mathbf{y}})}\left|\nabla\left(\hat{Q}-\hat{Q}_{s}\right)\right|^{2} d \mathbf{y} \leq & M_{15}\left(s^{3 \delta}+s^{2} r^{\delta}\right) \\
& -\int_{B_{s}(\overline{\mathbf{y}})} f_{b}(\hat{Q}) d \mathbf{y}+\frac{s}{m} \int_{\partial B_{s}(\overline{\mathbf{y}})} f_{b}(\hat{Q}) d \mathbf{s} .
\end{aligned}
$$


Here $m=m\left(r_{0}\right)$ such that $0<m<2$ and can be taken as close to 2 as desired provided $r_{0}$ is taken sufficiently small to begin with. Now set

$$
\gamma(s)=\int_{B_{s}(\overline{\mathbf{y}})} f_{b}(\hat{Q}) d \mathbf{y} .
$$

From (3.13) we have

$$
-M_{15}\left(s^{3 \delta}+s^{2} r^{\delta}\right) \leq-\gamma(s)+\frac{s}{m} \gamma^{\prime}(s)
$$

for almost every $0<s \leq r$. In what follows the constants $C_{i}$ will depend on $r, \delta$, and $\eta$. Let $0<\eta<\min (3 \delta, m)$. Dividing by $s^{1+\eta}$ and using the fact that $\gamma^{\prime}(s) \geq 0$ we find

$$
-M_{16} s^{\min (3 \delta, m)-\eta-1} \leq\left(\frac{\gamma(s)}{s^{\eta}}\right)^{\prime} .
$$

Since $\min (3 \delta, m)>\eta$ we can integrate this to obtain

$$
\frac{\gamma(s)}{s^{\eta}} \leq C_{1} \text { for } 0<s \leq r .
$$

Thus

$$
\int_{B_{s}(\overline{\mathbf{y}})} f_{b}(\hat{Q}) d \mathbf{y} \leq C_{1} s^{\eta} \quad \text { for } \quad|\overline{\mathbf{y}}| \leq r, \quad s \leq r,
$$

for $\eta<\min (3 \delta, m)$. We next get a corresponding, improved estimate for $|\nabla \hat{Q}|^{2}$. Since $\gamma(s)$ is absolutely continuous on $[0, r]$, given $0<t \leq r$ we can select $s_{0}$ so that $\frac{t}{2} \leq s_{0} \leq t$ and $t \gamma^{\prime}\left(s_{0}\right) \leq 2 \gamma(t)$. Then from (3.13) we get

$$
\int_{B_{s_{0}}(\overline{\mathbf{y}})} \mid \nabla\left(\hat{Q}-\left.\hat{Q}_{s_{0}}\right|^{2} d \mathbf{y} \leq C_{2}\left(t^{3 \delta}+t^{\eta}\right) \leq C_{3} t^{\eta} .\right.
$$

Since $\hat{Q}_{s_{0}}$ solves (3.3) and (3.4) we have that

$$
\int_{B_{\rho}(\overline{\mathbf{y}})}\left|\nabla \hat{Q}_{s_{0}}\right|^{2} d \mathbf{y} \leq C_{4}\left(\frac{\rho}{s_{0}}\right)^{2} \int_{B_{s_{0}}(\overline{\mathbf{y}})}\left|\nabla \hat{Q}_{s_{0}}\right|^{2} d \mathbf{y} \text { for } \rho \leq s_{0} .
$$

(See [6]; Ch. III.) Thus using (3.5) and (3.14)

$$
\int_{B_{\rho}(\overline{\mathbf{y}})}|\nabla \hat{Q}|^{2} d \mathbf{y} \leq C_{5}\left(\frac{\rho}{s_{0}}\right)^{2} \int_{B_{s_{0}}(\overline{\mathbf{y}})}|\nabla \hat{Q}|^{2} d \mathbf{y}+C_{6} t^{\eta} \text { for } \rho<s_{0},
$$

and it follows that

$$
\int_{B_{\rho}(\overline{\mathbf{y}})}|\nabla \hat{Q}|^{2} d \mathbf{y} \leq C_{7}\left(\frac{\rho}{t}\right)^{2} \int_{B_{t}(\overline{\mathbf{y}})}|\nabla \hat{Q}|^{2} d \mathbf{y}+C_{8} t^{\eta}
$$

for $0<\rho \leq t \leq r,|y| \leq r$, and $r \leq r_{0}$. Then from [6]; Ch. III, Lemma 2.1 we find that

$$
\int_{B_{t}(\overline{\mathbf{y}})}|\nabla \hat{Q}|^{2} d \mathbf{y} \leq C_{9} t^{\eta} \text { for }|\overline{\mathbf{y}}| \leq r, t \leq r .
$$

Given $0<\theta<1$ set $\delta_{1}=\min \left(\frac{5 \delta}{4}, \theta\right)$. We have strengthened (3.2) by showing that the inequality holds with $\delta$ replaced by $\delta_{1}$. The argument can be repeated a finite number of times to obtain the estimate with $\delta$ replaced by $\theta$. We return to (3.12) setting $\overline{\mathbf{y}}=\mathbf{o}, s=r$, and $\delta=\theta$. Using the definition of $\gamma(r)$ we find that there exists $r_{1}>0$ so that

$$
-2 M_{15} r^{3 \theta} \leq-\gamma(r)+\left(\frac{r}{2}+M_{8} r^{1+\theta}\right) \gamma^{\prime}(r)
$$


for almost every $0<r \leq r_{1}$. We need $\theta>\frac{2}{3}$ and set $\theta=\frac{3}{4}$. Multiplying (3.15) by the integrating factor $2 r^{-3}\left(1+2 M_{8} r^{3 / 4}\right)^{5 / 3}$ we obtain

$$
-M_{16} r^{-\frac{3}{4}} \leq\left[r^{-2}\left(1+2 M_{8} r^{3 / 4}\right)^{8 / 3} \gamma(r)\right]^{\prime} .
$$

Integrating from $r$ to $r_{1}$ we get

$$
r^{-2} \int_{B_{r}(\mathbf{o})} f_{b}(\hat{Q}) d \mathbf{y}=r^{-2} \gamma(r) \leq C=C\left(r_{1}\right)
$$

for $0<r \leq r_{1}$. We assumed that $\hat{Q}(\mathbf{o}) \in \partial \mathcal{M}$ implying that $\lim _{\mathbf{y} \rightarrow \mathbf{o}} f_{b}(\hat{Q}(\mathbf{y}))=\infty$ which is impossible.

We next apply the harmonic replacement method to a problem with its domain in $\mathbb{R}^{n}$.

Proof of Theorem 4 1. Let $B=B_{r}\left(\mathbf{x}_{0}\right) \subset \Omega \subset \mathbb{R}^{n}$ and let $u_{B}(\mathbf{x})$ be defined to have harmonic components and satisfy $u_{B}=u$ on $\partial B$. It follows from the maximum principle and the fact that $\mathcal{K}$ is convex that $u_{B}(\mathbf{x}) \in \overline{\mathcal{K}}$ for $\mathbf{x} \in B$. We can then compare $u$ and $u_{B}$,

$$
\int_{B}\left[\gamma|\nabla u|^{2}+\tilde{f}(u)\right] d \mathbf{x} \leq \int_{B}\left[\gamma\left|\nabla u_{B}\right|^{2}+\tilde{f}\left(u_{B}\right)\right] d \mathbf{x} .
$$

Since $u_{B}$ is harmonic we have that

$$
\gamma \int_{B}\left|\nabla\left(u-u_{B}\right)\right|^{2} d \mathbf{x}=\gamma \int_{B}\left(|\nabla u|^{2}-\left|\nabla u_{B}\right|^{2}\right) d \mathbf{x} .
$$

Using the fact that $\mathcal{K}$ is bounded and the Poincaré and Hölder inequalities we have that

$$
\begin{aligned}
\left.\int_{B} \kappa|| u\right|^{2}-\left|u_{B}\right|^{2} \mid d \mathbf{x} & \leq C_{0} \int_{B}\left|u-u_{B}\right| d \mathbf{x} \\
& \leq C_{1} r \int_{B}\left|\nabla\left(u-u_{B}\right)\right| d \mathbf{x} \\
& \leq \frac{\gamma}{2} \int_{B}\left|\nabla\left(u-u_{B}\right)\right|^{2} d \mathbf{x}+C_{2} r^{n+2} .
\end{aligned}
$$

Next, just as in the proof for Theorem 1 we have that

$$
\int_{B} f\left(u_{B}\right) d \mathbf{x} \leq \frac{r}{n} \int_{\partial B} f(u) d \mathbf{s} .
$$

Combining these facts with (3.16) we get

$$
-C_{3} r \leq \frac{d}{d r}\left(r^{-n} \int_{B_{r}\left(\mathbf{x}_{0}\right)} f(u) d \mathbf{s}\right) .
$$

Given $\delta>0$ set $\Omega_{\delta}=\{\mathbf{x} \in \Omega: \operatorname{dist}(\mathbf{x}, \partial \Omega) \geq \delta\}$. Then just as in the Proof of Theorem 3 we find that for $\mathbf{x} \in \Omega_{\delta}$ and $r<\delta$ that

$$
r^{-n} \int_{B_{r}(\mathbf{x})} f(u) d \mathbf{x} \leq \delta^{-n} \int_{B_{\delta}(\mathbf{x})} f(u) d \mathbf{x}+C_{4} \leq C_{5}<\infty
$$

where $C_{4}=C_{4}(\gamma, \delta)$ and $C_{5}=C_{5}\left(\gamma, \delta, \int_{\Omega} f(u) d \mathbf{x}\right)$. It follows that $f(u(\mathbf{x})) \leq C_{5}$ almost everywhere on $\Omega_{\delta}$. From the structure of $\tilde{f}$ we see that there is a constant $\beta>0$, $\beta=\beta\left(C_{5}\right)$ so that

$$
u(\mathbf{x}) \in \mathcal{K}_{\beta}=\{\mathbf{p} \in \mathcal{K}: \operatorname{dist}(\mathbf{p}, \partial \mathcal{K}) \geq \beta\}
$$


for almost every $\mathbf{x} \in \Omega_{\delta}$. Due to this we can take smooth variations with compact support about $u$ for $\mathcal{F}_{1}$ and as a consequence $u$ satisfies the energy's Euler-Lagrange equation. Since $\tilde{f}(\cdot) \in C^{\infty}(\mathcal{K})$ it follows that $\Delta u \in L_{\text {loc }}^{\infty}(\Omega)$ and we have that $u \in C^{1, \alpha}(\Omega)$ for $0<\alpha<1$. Higher regularity follows from elliptic estimates.

2. Assume now that $u=u_{0}$ on $\partial \Omega$. Let $r_{1}$ be a positive constant such that for each point $\mathbf{o}$ on $\partial \Omega, B_{r_{1}}(\mathbf{o}) \cap \Omega$ is a coordinate neighborhood. We first estimate $r^{-n} \int_{B_{r}(\mathbf{o}) \cap \Omega} f(u) d \mathbf{x}$ for $\mathbf{o} \in \partial \Omega$ and $0<r<r_{1}$. Fix $\mathbf{o}$ and $r$. Set $B=B_{r}(\mathbf{o})$ and let $u_{B}$ have harmonic components in $B \cap \Omega$ with $u_{B}=u$ on $\partial(B \cap \Omega)$. Then just as above we have

$$
\begin{aligned}
& \frac{\gamma}{2} \int_{B \cap \Omega}\left|\nabla\left(u-u_{B}\right)\right|^{2} d \mathbf{x}+\int_{B \cap \Omega} f(u) d \mathbf{x} \\
& \quad \leq \int_{B \cap \Omega} f\left(u_{B}\right) d \mathbf{x}+C_{1} r^{n+2} .
\end{aligned}
$$

Set $f_{\mathbf{o}}(z)=f(z)-D_{u} f(u(\mathbf{o})) \cdot(z-u(\mathbf{o}))-f(u(\mathbf{o}))$. From (3.18) we have

$$
\begin{aligned}
& \frac{\gamma}{2} \int_{B \cap \Omega}\left|\nabla\left(u-u_{B}\right)\right|^{2} d \mathbf{x}+\int_{B \cap \Omega} f_{\mathbf{o}}(u) d \mathbf{x} \\
& \quad \leq \int_{B \cap \Omega} f_{\mathbf{o}}\left(u_{B}\right) d \mathbf{x}-\int_{B \cap \Omega} D_{u} f(u(\mathbf{o})) \cdot\left(u-u_{B}\right) d \mathbf{x}+C_{1} r^{n+2} .
\end{aligned}
$$

Note that since $u_{0} \in C^{\sigma}(\partial \Omega ; \mathcal{K})$ we have $u_{0}(\partial \Omega) \subset \mathcal{K}_{\beta}$ for some $\beta>0$ and as such $u_{0}$ is separated from $\partial \mathcal{K}$. It then follows that there is a constant $C_{2}$ such that $\left|D_{u} f(u(\mathbf{o}))\right|+|f(u(\mathbf{o}))| \leq C_{2}$ for all $\mathbf{o} \in \partial \Omega$. Thus we have

$$
\int_{B \cap \Omega} f_{\mathbf{0}}(u) d \mathbf{x} \leq \int_{B \cap \Omega} f_{\mathbf{0}}\left(u_{B}\right) d \mathbf{x}+C_{3} r^{n+2} .
$$

Let $w(\mathbf{x})$ satisfy $\Delta w=1$ in $B \bigcap \Omega$ and $w=0$ on $\partial(B \bigcap \Omega)$. Since $f_{\mathbf{o}}$ is convex we have

$$
\int_{B \cap \Omega} f_{\mathbf{o}}\left(u_{B}\right) d \mathbf{x} \leq \int_{\partial(B \cap \Omega)} f_{\mathbf{o}}\left(u_{B}\right) \partial_{\nu} w d \mathbf{s} .
$$

Using barriers we have $\left|\partial_{\nu} w\right| \leq C_{3} r$ on $\partial(B \cap \Omega)$. Since $\frac{1}{2 n}\left(|\mathbf{x}-\mathbf{o}|^{2}-r^{2}\right) \leq w$ we have the specific estimate $\partial_{\nu} w \leq \frac{r}{n}$ on $\Omega \bigcap \partial B$. Using that $f_{\mathbf{o}}(\cdot) \geq 0$ we get

$$
\int_{\partial(B \cap \Omega)} f_{\mathbf{o}}\left(u_{B}\right) \partial_{\nu} w d \mathbf{s} \leq \frac{r}{n} \int_{\Omega \cap \partial B} f_{\mathbf{o}}(u) d \mathbf{s}+C_{4} r \int_{B \cap \partial \Omega} f_{\mathbf{o}}\left(u_{0}\right) d \mathbf{s} .
$$

We have that $f_{\mathbf{o}}(\cdot)$ is smooth on $u_{0}(\partial \Omega), f_{\mathbf{o}}\left(u_{0}(\mathbf{o})\right)=0, D_{u} f_{\mathbf{o}}\left(u_{0}(\mathbf{o})\right)=0$, and $u_{0} \in$ $C^{\sigma}(\partial \Omega)$. Thus

$$
r \int_{B \cap \partial \Omega} f_{\mathbf{o}}\left(u_{0}\right) d \mathbf{s} \leq C_{5} r \int_{B \cap \partial \Omega}\left|u_{0}(\mathbf{x})-u_{0}(\mathbf{o})\right|^{2} d \mathbf{s} \leq C_{6} r^{n+2 \sigma} .
$$

We arrive at

$$
\int_{\Omega \cap B_{r}(\mathbf{o})} f_{\mathbf{o}}(u) d \mathbf{x} \leq \frac{r}{n} \int_{\Omega \bigcap \partial B_{r}(\mathbf{o})} f_{\mathbf{o}}(u) d \mathbf{s}+C_{7} r^{n+2 \sigma} .
$$

Multiplying the inequality by $\frac{n}{r^{n+1}}$ and integrating we get

$$
r^{-n} \int_{\Omega \cap B_{r}(\mathbf{o})} f_{\mathbf{o}}(u) d \mathbf{x} \leq r_{1}^{-n} \int_{\Omega \cap B_{r_{1}}(\mathbf{o})} f_{\mathbf{o}}(u) d \mathbf{x}+C_{8} \text { for } r \leq r_{1} .
$$


It follows that

$$
\begin{aligned}
r^{-n} \int_{\Omega \cap B_{r}(\mathbf{o})} f(u) d \mathbf{x} & \leq r_{1}{ }^{-n} \int_{\Omega \cap B_{r_{1}}(\mathbf{o})} f(u) d \mathbf{x}+C_{9} \\
& \leq C_{10} \quad \text { for } r \leq r_{1} .
\end{aligned}
$$

We can now prove that $f(u)$ is bounded on $\Omega$. Indeed if $\mathbf{x} \in \Omega_{\frac{r_{1}}{2}}$ then from (3.17) we have $f(u(\mathbf{x})) \leq C_{5}$. If $\mathbf{x} \in \Omega \backslash \Omega_{\frac{r_{1}}{2}}$ then $|\mathbf{x}-\mathbf{o}|=\operatorname{dist}(\mathbf{x}, \partial \Omega) \leq \frac{r_{1}}{2}$ for some $\mathbf{o} \in \partial \Omega$. Set $\frac{r}{2}=|\mathbf{x}-\mathbf{o}|$. From (3.17) and (3.19), for $\rho<\frac{r}{2}$ it holds that

$$
\begin{aligned}
\rho^{-n} \int_{B_{\rho}(\mathbf{x})} f(u) d \mathbf{x} & \leq\left(\frac{r}{2}\right)^{-n} \int_{B_{\frac{r}{2}}(\mathbf{x})} f(u) d \mathbf{x}+C_{4} \\
& \leq 2^{n} r^{-n} \int_{\Omega \cap B_{r}(\mathbf{o})} f(u) d \mathbf{x}+C_{4} \leq C_{11}
\end{aligned}
$$

and we find that $f(u(\mathbf{x})) \leq C_{11}$. It follows as above that $u(\bar{\Omega}) \subset \mathcal{K}_{\beta}$ for some $\beta>0$. We have that $\partial \Omega$ is $C^{2, \sigma}, u_{0} \in C^{\sigma}$ and $\Delta u \in L^{\infty}(\Omega)$. It holds that $u \in C^{\sigma}(\bar{\Omega})$. Indeed, we write $u=h+z$ where $h$ has harmonic components and $h=u_{0}$ on $\partial \Omega$. It follows from elliptic $L^{p}$ estimates that $z \in C^{1, \alpha}(\bar{\Omega})$ for each $0<\alpha<1$ and from Schauder theory that $h \in C^{\sigma}(\bar{\Omega})$. See [1], Theorem 9.3.

We can apply Theorem 4 to tensor-valued functions as in Sect. 1 with $f_{b}$ satisfying (1.2)-(1.3). Let $[A]^{S T}$ denote the symmetric and traceless part of the tensor $A$.

Corollary 3 Let $D$ be a bounded domain in $\mathbb{R}^{3}$ and let $Q \in H^{1}(D ; \overline{\mathcal{M}})$ be a local minimizer for $\mathcal{F}(V ; D)=\int_{D}\left[L_{1}|\nabla V|^{2}+f_{b}(V)\right] d \mathbf{x}$ such that $\mathcal{F}(Q ; D)<\infty$. Then $Q \in C^{\infty}(D)$, $Q(\mathbf{x}) \in \mathcal{M}$ for each $\mathbf{x} \in D$ and

$$
2 L_{1} \Delta Q(\mathbf{x})=\left[D_{Q} f_{b}(Q(\mathbf{x}))\right]^{S T} \text { in } D .
$$

Proof Let $\left\{E_{1}, \ldots, E_{5}\right\}$ be an orthonormal basis for $\mathcal{S}_{0}$ and parameterize $W \in \mathcal{S}_{0}$ by $W(u)=$ $\sum_{i=1}^{5} u_{i} E_{i}$ for $u \in \mathbb{R}^{5}$. Then $\mathcal{K}:=\left\{u \in \mathbb{R}^{5}: W(u) \in \mathcal{M}\right\}$ is a bounded, open, and convex. For $V \in H^{1}(D ; \overline{\mathcal{M}})$ we can write

$$
L_{1}|\nabla V(\mathbf{x})|^{2}+f_{b}(V(\mathbf{x}))=L_{1}|\nabla u(\mathbf{x})|^{2}+f_{b}(V(u(\mathbf{x})))
$$

and the assertions follow from Theorem 4.

In the same manner we have the following result for the Dirichlet problem.

Corollary 4 Assume $D \subset \mathbb{R}^{3}$ is a bounded domain with a $C^{2, \sigma}$ boundary and $Q_{0} \in$ $H^{1 / 2}(\partial D ; \overline{\mathcal{M}}) \cap C^{\sigma}(\partial D ; \mathcal{M})$ for some $0<\sigma<1$. If $Q$ is a minimizer for $\mathcal{F}(V ; D)=$ $\int_{D}\left[L_{1}|\nabla V|^{2}+f_{b}(V)\right] d \mathbf{x}$ in $H^{1}(D ; \overline{\mathcal{M}})$ subject to $Q=Q_{0}$ on $\partial D$ then $Q(D) \subset \subset \mathcal{M}$ and $Q \in C^{\sigma}(\bar{D} ; \mathcal{M})$.

The last result was first proved by Ball-Majumdar in [2]. Specifically they worked with $Q_{0} \in H^{1 / 2}(\partial D ; \overline{\mathcal{M}})$ that is valued in a compact subset of $\mathcal{M}$ and proved that $Q(D) \subset \subset \mathcal{M}$.

Open Access This article is distributed under the terms of the Creative Commons Attribution 4.0 International License (http://creativecommons.org/licenses/by/4.0/), which permits unrestricted use, distribution, and reproduction in any medium, provided you give appropriate credit to the original author(s) and the source, provide a link to the Creative Commons license, and indicate if changes were made. 


\section{Appendix 1}

In this section we derive a reverse Hölder inequality that when combined with the proof from [6]; Ch. VI, Proposition 1 demonstrates that local minimizers are regular near $\partial \Omega \cap \Lambda(Q)$. Let $\mathbf{x}_{0} \in \partial \Omega \backslash \Lambda(Q)$. Fix $r>0$ so that $\overline{B_{4 r}}\left(\mathbf{x}_{0}\right) \cap \Lambda(Q)=\emptyset$ and take $r$ sufficiently small so that $B_{4 r}\left(\mathbf{x}_{0}\right) \cap \Omega$ is diffeomorphic to a half-disk. From what has been proved we can assume that $Q=Q(\mathbf{z})$ as in (1.6), where $\mathbf{z} \in C^{2}\left(B_{2 r}\left(\mathbf{x}_{0}\right) \cap \Omega\right) \cap C^{\sigma}\left(B_{2 r}\left(\mathbf{x}_{0}\right) \cap \bar{\Omega}\right) \cap H^{1}\left(B_{2 r}\left(\mathbf{x}_{0}\right) \cap \Omega\right)$ satisfying the Euler-Lagrange equation for $\mathcal{F}^{\prime}(\cdot)$,

$$
\begin{aligned}
\partial_{x_{k}}\left(A_{i j}^{l k}(\mathbf{z}) \partial_{x_{l}} z^{i}\right) & +B_{i m j}^{k l}(\mathbf{z}) \partial_{x_{l}} z^{i} \partial_{x_{k}} z^{m}+C_{i j}^{l}(\mathbf{z}) \partial_{x_{l}} z^{i} \\
& +D_{j}(\mathbf{z})=0 \quad \text { in } \quad B_{2 r}\left(\mathbf{x}_{0}\right) \cap \Omega,
\end{aligned}
$$

with boundary condition

$$
\mathbf{z}=\mathbf{z}_{0} \quad \text { on } \quad B_{2 r}\left(\mathbf{x}_{0}\right) \cap \partial \Omega .
$$

Here the coefficients are $C^{1}$ functions on a neighborhood $\mathcal{K}$ of the range of $\left.\mathbf{z}\right|_{B_{2 r}\left(\mathbf{x}_{0}\right) \cap \Omega}$, such that

$$
\|\mathbf{A}\|_{C^{1}(\overline{\mathcal{K}})},\|\mathbf{B}\|_{C^{1}(\overline{\mathcal{K}})},\|\mathbf{C}\|_{C^{1}(\overline{\mathcal{K}})},\|\mathbf{D}\|_{C^{1}(\overline{\mathcal{K}})} \leq M<\infty .
$$

For $\lambda>0$ we assume that

$$
A_{i j}^{l k}(\mathbf{z}) \zeta_{l}^{i} \zeta_{k}^{j} \geq \lambda|\zeta|^{2} \text { for } \zeta \in \mathbb{R}^{5 \times 2} \text { and } \quad \mathbf{z} \in \overline{\mathcal{K}},
$$

and $\mathbf{z}_{0} \in C^{2}(\partial \Omega)$. Set

$$
\delta=\sup \left\|\mathbf{z}-\mathbf{z}_{0}\right\|_{C\left(B_{2 r}\left(\mathbf{x}_{0}\right) \cap \Omega\right)} .
$$

We prove

Proposition 1 There exist constants $\delta_{0}, C>0$ depending on $\lambda, M, \mathbf{z}_{0}$, and $\Omega$ so that if $\delta \leq \delta_{0}$ then $D^{2} \mathbf{z},|D \mathbf{z}|^{2} \in L^{2}\left(B_{r}\left(\mathbf{x}_{0}\right) \cap \Omega\right)$ and for $0<\rho \leq \frac{r}{2}$

$$
f_{B_{\rho}\left(\mathbf{x}_{0}\right) \cap \Omega}\left[1+\left|D^{2} \mathbf{z}\right|+|D \mathbf{z}|^{2}\right]^{2} d \mathbf{x} \leq C\left(f_{B_{2 \rho}\left(\mathbf{x}_{0}\right) \cap \Omega}\left[1+\left|D^{2} \mathbf{z}\right|+|D \mathbf{z}|^{2}\right] d \mathbf{x}\right)^{2} .
$$

Proof. For simplicity we assume that $\mathbf{x}_{0}=\mathbf{0}=(0,0)$ and

$$
B_{2 r}(\mathbf{o}) \cap \Omega=B_{2 r}^{+}=\left\{\left(x_{1}, x_{2}\right):|\mathbf{x}|<2 r, x_{2}>0\right\},
$$

such that

$$
B_{2 r}(\mathbf{o}) \cap \partial \Omega=\left\{\left(x_{1}, 0\right):\left|x_{1}\right|<2 r\right\} .
$$

The general problem reduces to this case by flattening the boundary. This introduces an explicit $\mathbf{x}$-dependence for the coefficients in (4.1) that does not affect the estimates; however the constants $C_{i}$ appearing below will also depend on $\Omega$.

Let $\eta \in C_{c}^{\infty}\left(B_{2 r}(\mathbf{o})\right)$ such that $\eta=1$ on $B_{r}(\mathbf{o})$. Let $h \neq 0$ and set

$$
\Delta_{1}^{h} u(\mathbf{x})=\frac{1}{h}\left[u\left(x_{1}+h, x_{2}\right)-u\left(x_{1}, x_{2}\right)\right] .
$$

We multiply (4.1) by the test functions $\Delta_{1}^{-h}\left[\eta^{4} \Delta_{1}^{h}\left(\mathbf{z}-\mathbf{z}_{0}\right)\right]$ and $\left(\mathbf{z}-\mathbf{z}_{0}\right)\left|\Delta_{1}^{h}\left(\mathbf{z}-\mathbf{z}_{0}\right)\right|^{2} \eta^{4}$. Integrating over $B_{2 r}^{+}$in each case yields for $\delta \leq \delta_{0}$

$$
\int_{B_{2 r}^{+}}\left[\left|\Delta_{1}^{h} D \mathbf{z}\right|^{2}+\left|\Delta_{1}^{h} \mathbf{z}\right|^{2}|D \mathbf{z}|^{2}\right] \eta^{4} d \mathbf{x}
$$




$$
\begin{aligned}
\leq & C_{1} \int_{B_{2 r}^{+}}\left[1+|D \mathbf{z}|^{2}+\left|\Delta_{1}^{h} \mathbf{z}\right|^{2}\right] \eta^{4} d \mathbf{x} \\
& +C_{2} \int_{B_{2 r}^{+}}\left|\Delta_{1}^{h}\left(\mathbf{z}-\mathbf{z}_{0}\right)\right|^{2}|D \eta|^{2} \eta^{2} d \mathbf{x}
\end{aligned}
$$

where the $C_{j}$ depend only on $\delta_{0}, \lambda_{1}, M$, and $\left\|\mathbf{z}_{0}\right\|_{C^{2}}$. Letting $h \rightarrow 0$ gives

$$
\begin{aligned}
& \int_{B_{2 r}^{+}}\left[\left|\partial_{x_{1}} D \mathbf{z}\right|^{2}+\left|\partial_{x_{1}} \mathbf{z}\right|^{4} \eta^{4} d \mathbf{x}\right. \\
& \leq C_{3} \int_{B_{2 r}^{+}}\left[1+|D \mathbf{z}|^{2}\right] \eta^{4} d \mathbf{x} \\
& \quad+C_{4} \int_{B_{2 r}^{+}}\left|\partial_{x_{1}}\left(\mathbf{z}-\mathbf{z}_{0}\right)\right|^{2}|D \eta|^{2} \eta^{2} d \mathbf{x} .
\end{aligned}
$$

We next estimate $\left|\partial_{x_{2}}^{2} \mathbf{z}\right|^{2} \eta^{4}$ and $\left|\partial_{x_{2}} \mathbf{z}\right|^{4} \eta^{4}$. From (4.1) we have the pointwise bound

$$
\left|\partial_{x_{2}}^{2} \mathbf{z}\right|^{2} \leq C_{5}\left(\left|\partial_{x_{2}} \mathbf{z}\right|^{4}+\left|\partial_{x_{1}} D \mathbf{z}\right|^{2}+\left|\partial_{x_{1}} \mathbf{z}\right|^{4}+1\right) .
$$

For $\varepsilon>0$ set $B_{\varepsilon, r}^{+}=B_{r}^{+} \cap\left\{x_{2}>\varepsilon\right\}$. Multiplying (4.1) by $\left(\mathbf{z}-\mathbf{z}_{0}\right)\left|\partial_{x_{2}} \mathbf{z}\right|^{2} \eta^{4}$, integrating over $B_{\varepsilon, 2 r}^{+}$with $\delta<\delta_{0}$ yields

$$
\begin{aligned}
\int_{B_{\varepsilon, 2 r}^{+}}\left|\partial_{x_{2}} \mathbf{z}\right|^{4} \eta^{4} d \mathbf{x} \leq & C_{6} \delta \int_{B_{\varepsilon, 2 r}^{+}}\left|\partial_{x_{2}}^{2} \mathbf{z}\right|^{2} \eta^{4} d \mathbf{x} \\
& +C_{7} \int_{\left\{x_{2}=\varepsilon\right\}}\left|\mathbf{z}-\mathbf{z}_{0} \| D \mathbf{z}\right|^{3} \eta^{4} d x_{1} \\
& +C_{8} \int_{B_{\varepsilon, 2 r}^{+}}\left[\left|\partial_{x_{1}} D \mathbf{z}\right|^{2}+\left|\partial_{x_{1}} \mathbf{z}\right|^{4}+1\right] \eta^{4} d \mathbf{x} \\
& +C_{9} \int_{B_{\varepsilon, 2 r}^{+}}\left|\mathbf{z}-\mathbf{z}_{0}\right|^{4}|D \eta|^{4} d \mathbf{x} .
\end{aligned}
$$

We next estimate

$$
\begin{aligned}
\int_{\left\{x_{2}=\varepsilon\right\}}|D \mathbf{z}|^{3} \eta^{4} d x_{1} \leq & \int_{B_{\varepsilon, 2 r}^{+}}\left|\partial_{x_{2}}\left(|D \mathbf{z}|^{3} \eta^{4}\right)\right| d \mathbf{x} \\
\leq & C_{10} \int_{B_{\varepsilon, 2 r}^{+}}\left[\left|\partial_{x_{2}} D \mathbf{z}\right|^{2}+|D \mathbf{z}|^{4}\right] \eta^{4} d \mathbf{x} \\
& +C_{11} \int_{B_{\varepsilon, 2 r}^{+}}|D \eta|^{4} d \mathbf{x}
\end{aligned}
$$

We use this to estimate the second term on the right side of (4.4). This together with the integral of (4.3) times $\eta^{4}$ over $B_{\varepsilon, 2 r^{+}}$and (4.2) for $\delta \leq \delta_{0}$ yields

$$
\int_{B_{\varepsilon, 2 r}^{+}}\left[\left|D^{2} \mathbf{z}\right|^{2}+|D \mathbf{z}|^{4}\right] \eta^{4} d \mathbf{x} \leq E<\infty
$$

where $E$ is independent of $\varepsilon$. From this point we proceed using the fact that $\left|D^{2} \mathbf{z}\right|+|D \mathbf{z}|^{2} \in$ $L^{2}\left(B_{r}^{+}\right)$and replacing $\eta$ by $\tilde{\eta} \in C^{\infty}\left(B_{2 \rho}(\mathbf{o})\right)$ such that $\tilde{\eta}=1$ on $B_{\rho}(\mathbf{o})$ for $\rho \leq \frac{r}{2}$. Since 
$\lim _{\varepsilon \rightarrow 0}\left|\mathbf{z}\left(x_{1}, \varepsilon\right)-\mathbf{z}_{0}\left(x_{1}\right)\right|=0$ uniformly in $x_{1}$ we now have

$$
\lim _{\varepsilon \rightarrow 0} \int_{\left\{x_{2}=\varepsilon\right\}}\left|\mathbf{z}-\mathbf{z}_{0}\right||D \mathbf{z}|^{3} \tilde{\eta}^{4} d x_{1}=0 .
$$

Using this, (4.2), (4.3), and (4.4) give

$$
\begin{aligned}
& \int_{B_{2 \rho}^{+}}\left[\left|D^{2} \mathbf{z}\right|^{2}+|D \mathbf{z}|^{4}\right] \tilde{\eta}^{4} d \mathbf{x} \\
& \leq C_{12} \int_{B_{2 \rho}^{+}}\left[1+|D \mathbf{z}|^{2}\right] \tilde{\eta}^{4} d \mathbf{x} \\
& \quad+C_{13} \int_{B_{2 \rho}^{+}}\left|\partial_{x_{1}}\left(\mathbf{z}-\mathbf{z}_{0}\right)\right|^{2}|D \tilde{\eta}|^{2} \tilde{\eta}^{2} d \mathbf{x} \\
& \quad+C_{14} \int_{B_{2 \rho}^{+}}\left|\mathbf{z}-\mathbf{z}_{0}\right|^{4}|D \tilde{\eta}|^{4} d \mathbf{x} .
\end{aligned}
$$

We can assume that $|D \tilde{\eta}| \leq \frac{C}{\rho}$. Since $\mathbf{z}-\mathbf{z}_{0}$ and $\partial_{x_{1}}\left(\mathbf{z}-\mathbf{z}_{0}\right)$ vanish for $x_{2}=0$ we can apply the Sobolev-Poincaré inequality to the last two terms. We get

$$
\int_{B_{2 \rho}^{+}}\left|\partial_{x_{1}}\left(\mathbf{z}-\mathbf{z}_{0}\right)\right|^{2} d \mathbf{x} \leq C_{15}\left(\int_{B_{2 \rho}^{+}}\left|D^{2}\left(\mathbf{z}-\mathbf{z}_{0}\right)\right| d \mathbf{x}\right)^{2},
$$

and

$$
\rho^{-2} \int_{B_{2 \rho}^{+}}\left|\mathbf{z}-\mathbf{z}_{0}\right|^{4} d \mathbf{x} \leq C_{16}\left(\int_{B_{2 \rho}^{+}}\left|D\left(\mathbf{z}-\mathbf{z}_{0}\right)\right|^{2} d \mathbf{x}\right)^{2} .
$$

Inserting these estimates into (4.6) gives our result.

\section{References}

1. Agmon, S., Douglis, A., Nirenberg, L.: Estimates near the boundary for solutions of elliptic partial differential equations satisfying general boundary conditions. I. Comm. Pure Appl. Math. vol. 12, pp. 623727 (1959)

2. Ball, J.M., Majumdar, A.: Nematic liquid crystals; from Maier-Saupe to a continuum theory. Mol. Cryst. Liq. Cryst. 525(1), 1-11 (2010)

3. Evans, L., Gareipy, R.: Measure Theory and Fine Properties of Functions. CRC Press, Boca Raton (1992)

4. Evans, L., Kneuss, L.O., Tran, H.: Partial regularity for minimizers of singular energy functionals, with application to liquid crystal models (2014) arXiv:1312.4471

5. Feireisl, E., Rocca, E., Schimperna, G., and Zarnescu, A.: Nonisothermal nematic liquid crystal flows with the Ball-Majumdar free energy. Ann. Mat. Pura Appl. (4) 194(5):1269-1299 (2015)

6. Giaquinta, M.: Multiple integrals in the calculus of variations in nonlinear elliptic systems. Ann. Math. Stud. 105 Princeton University Press (1983)

7. Katriel, J., Kventsel, G.F., Luckhurst, G.R., Sluckin, T.J.: Free energies in the Landau and molecular field approaches. Liq. Cryst. Liq. Cryst. 1, 337-355 (1986)

8. Longa, L., Monselesan, D., Trebin, H.R.: An extension of the Landau de Gennes theory for liquid crystals. Liq. Cryst. 2(6), 769-796 (1987)

9. Maier, W., Saupe, A.: A simple molecular statistical theory of the nematic liquid-crystalline phase. I.Z. Maturf. a.14:882-889 (1959)

10. Morrey, C.B. Jr.: Second order elliptic systems of differential equations. Ann. Math. Stud. No. 33, Princeton University Press, pp. 101-159 (1954) 